\title{
Towards exotic matter and discrete non-abelian symmetries in F-theory
}

\author{
Mirjam Cvetič, ${ }^{a, b}$ Jonathan J. Heckman ${ }^{a}$ and Ling Lin $^{a}$ \\ ${ }^{a}$ Department of Physics and Astronomy, University of Pennsylvania, \\ Philadelphia, PA 19104, U.S.A. \\ ${ }^{b}$ Center for Applied Mathematics and Theoretical Physics, \\ University of Maribor, Maribor, Slovenia \\ E-mail: cvetic@physics.upenn.edu, jheckman@sas.upenn.edu, \\ lling@physics.upenn.edu
}

ABSTRACT: We present a prescription in F-theory for realizing matter in "exotic" representations of product gauge groups. For $6 \mathrm{D}$ vacua, bifundamental hypermultiplets are engineered by starting at a singular point in moduli space which includes $6 \mathrm{D}$ superconformal field theories coupled to gravity. A deformation in Higgs branch moduli space takes us to a weakly coupled gauge theory description. In the corresponding elliptically fibered Calabi-Yau threefold, the minimal Weierstrass model parameters $(f, g, \Delta)$ vanish at collisions of the discriminant at least to order $(4,6,12)$, but with sufficiently high order of tangency to ensure the existence of T-brane deformations to a weakly coupled gauge theory with exotic bifundamentals. We present explicit examples including bifundamental hypermultiplets of $\mathfrak{e}_{7} \times \mathfrak{s u}_{2}$ and $\mathfrak{e}_{6} \times \mathfrak{s u}_{3}$, each of which have dual heterotic orbifold descriptions. Geometrically, these matter fields are delocalized across multiple points of an F-theory geometry. Symmetry breaking with such representations can be used to produce high dimension representations of simple gauge groups such as the four-index symmetric representation of $\mathfrak{s u}_{2}$ and the three-index symmetric representation of $\mathfrak{s u}_{3}$, and after further higgsing can yield discrete non-abelian symmetries.

KEywords: F-Theory, String Duality, Superstrings and Heterotic Strings

ARXIV EPRINT: 1806.10594 


\section{Contents}

1 Introduction 1

2 Prescription for exotic bifundamentals 5

3 Engineering the $(56,2)$ of $\mathfrak{e}_{7} \times \mathfrak{s u}_{2} \quad 7$

3.1 T-branes and heterotic orbifolds 8

$\begin{array}{llr}3.2 & \text { Generic Weierstrass model with } \mathfrak{e}_{7} \times \mathfrak{s u}_{2} & 9\end{array}$

$\begin{array}{lll}3.3 & \text { Orbifold conditions from heterotic/F-theory duality } & 12\end{array}$

$\begin{array}{ll}3.4 & \text { The strongly coupled sector of the global model } \\ 3.5 & 13\end{array}$

$\begin{array}{ll}3.5 & \text { Adding more bifundamentals } \\ \end{array}$

4 Engineering the $(27,3)$ of $\mathfrak{e}_{6} \times \mathfrak{s u}_{3} \quad 17$

$\begin{array}{ll}\text { 4.1 Tangency of intersection and the need for a Type IV fiber } & 19\end{array}$

$\begin{array}{ll}4.2 & \text { Enhancing the strongly coupled sector } 20\end{array}$

4.3 Engineering the $\mathfrak{u}(1)$ and singlet counting 22

$\begin{array}{ll}4.4 & \text { Adding more bifundamentals } \\ \end{array}$

5 Breaking patterns $\quad 26$

5.1 The $(\mathbf{5 6}, \mathbf{2})$ of $\mathfrak{e}_{7} \times \mathfrak{s u}_{2} \quad 27$

5.2 The $(\mathbf{2 7}, \mathbf{3})$ of $\mathfrak{e}_{6} \times \mathfrak{s u}_{3} \quad 28$

$\begin{array}{llr}6 & \text { Conclusions } & 29\end{array}$

$\begin{array}{lll}\text { A Tate model with } \mathfrak{e}_{6} \times \mathfrak{s u}_{3} & 30\end{array}$

B Branching rules for bifundamental multiplets $\quad 32$

$\begin{array}{lll}\text { B.1 } \mathfrak{e}_{7} \times \mathfrak{s u}_{2} & 32\end{array}$

B.2 $\mathfrak{e}_{6} \times \mathfrak{s u}_{3}$

\section{Introduction}

One of the important lessons from string theory is that not all quantum field theories can be consistently coupled to quantum gravity. From a bottom up perspective, string theory can be viewed as pointing the way to subtle consistency conditions which might otherwise be missed. With this in mind, it is clearly important to delineate the full space of quantum field theories which can be realized in string theory [1,2].

For example, unlike from a purely effective field theory perspective, there seem to be clear limitations on the matter content in weakly coupled (supersymmetric) gauge theories that are realized in string compactifications. The most straightforward examples in 
perturbative open string theories involve matter in various two-index representations of the classical gauge groups $\mathrm{U}(N), \mathrm{SO}(N), \mathrm{Sp}(N)$. This can be widened in F-theory [3-5] to include all the exceptional groups, but again, matter fields tend to transform in low dimension representations (namely the fundamental or small extensions thereof) such as the $\mathbf{2 7}$ of $E_{6}$.

It is sometimes possible to arrange for more exotic representations in various free fermion constructions, as for example with heterotic strings at higher Kac-Moody levels (see, e.g., [6] and references therein), but even here, there are limitations to what can be obtained. The question of which $6 \mathrm{D}$ supergravity models can be embedded in F-theory has been the subject of extensive research [7-13].

A related but far less explored question has to do with the structure of possible discrete gauge groups which can be consistently realized. There are by now various examples of models with abelian symmetries, but even in this case the order of the group is typically quite small. Even fewer examples are available for non-abelian discrete groups. The latter are of interest in their own right, and also have applications to topological field theory and particle physics. Some work on the stringy realization of discrete non-abelian groups includes [14-19] as well as [20] in F-theory. As of this writing there does not appear to be a systematic method to determine whether a particular finite group can appear.

In a certain sense, the appearance of matter in high dimension representations of continuous gauge groups and non-abelian discrete gauge groups are related since the latter can be obtained by giving vevs to scalars transforming in high dimension representations [21]. From this perspective, it is natural to ask whether there are systematic ways to find compactifications which contain such structures.

Part of the issue with realizing higher dimension representations is that the standard way to engineer low energy effective field theories via string compactification typically breaks a substantial part of the higher-dimensional gauge group, leaving only a lower rank remnant behind. For example, in the context of compactifications of the heterotic string, one often specifies a vector bundle with structure group $K \subset E_{8}$. The commutant subgroup $G$ is then all that survives in the low energy theory. This in turn limits the matter content $G$ to low dimension representations. Related behavior occurs in F-theory and M-theory models, where the appearance of a non-trivial vector bundle is instead signalled by the presence of a generic unfolding of a singularity.

Tuning the moduli of a compactification provides one way to realize such exotica. For example, it has been known since the early days of heterotic strings compactified on orbifolds [22, 23], that it is possible to achieve matter fields in the $(\mathbf{5 6}, \mathbf{2})$ of $\mathfrak{e}_{7} \times \mathfrak{s u} \mathfrak{u}_{2}$ as well as the $(\mathbf{2 7}, \mathbf{3})$ of $\mathfrak{e}_{6} \times \mathfrak{s u}_{3}$ (see e.g. [24] for this and related examples). Perhaps surprisingly, in F-theory the corresponding geometric realization of these exotic bifundamental matter fields has only been studied in a few cases (see, e.g., [25]). Other examples with exotic matter representations in F-theory involve matter on a singular divisor [12, 26-28]. As far as we are aware, however, there is no systematic "rule of thumb" for how to realize such matter representations in F-theory.

With this in mind, our aim in this work will be to develop a set of geometric criteria for engineering exotic matter field representations in F-theory. In field theory terms, our 
main interest will be on $6 \mathrm{D}$ theories in flat space with hypermultiplets in "exotic" representations $\left(\mathbf{R}_{1}, \mathbf{R}_{2}\right)$ of some gauge algebra $\mathfrak{g}_{1} \times \mathfrak{g}_{2}$. As already mentioned, activating vevs for these matter fields also provides a mechanism for generating examples of discrete non-abelian symmetries.

From a geometric perspective, we consider elliptically fibered Calabi-Yau threefolds $X \rightarrow B$ with minimal Weierstrass model:

$$
y^{2}=x^{3}+f x+g
$$

so that the vanishing locus for the discriminant $\Delta=4 f^{3}+27 g^{2}$ has components supporting gauge algebra $\mathfrak{g}_{1} \times \mathfrak{g}_{2}$. Matter in low-dimensional representations comes about from collisions of these components. We get weakly coupled matter when the multiplicity of vanishing for $(f, g, \Delta)$ is below $(4,6,12)$. We can also realize $6 \mathrm{D}$ superconformal field theories (SCFTs) when the multiplicity of vanishing is at least $(4,6,12)$ since then, the elliptic fiber does not remain in Kodaira-Tate form [4, 5, 29-31] (see [32] for a recent review of 6D SCFTs from a top down perspective). In this case, to truly understand the physics of the F-theory model, we need to perform blow-ups of the base. Collapsing the additional $\mathbb{P}^{1}$ 's down to zero size then takes us back to the SCFT point. An interesting open problem is to determine which $6 \mathrm{D}$ SCFTs can be consistently coupled to gravity. For some results in this direction, see e.g. [10, 33, 34].

Now, in the context of F-theory, when one attempts to use the standard Tate algorithm to engineer "exotic" high dimension representations $\left(\mathbf{R}_{1}, \mathbf{R}_{2}\right)$ of some gauge algebra $\mathfrak{g}_{1} \times \mathfrak{g}_{2}$, one inevitably finds that the multiplicity of vanishing for $(f, g, \Delta)$ is at least $(4,6,12)$, indicating the appearance of a 6D SCFT lurking at some point of moduli space.

But as has been appreciated for some time, the Weierstrass model provides only partial information on the effective field theory specified by an F-theory model. To remove possible ambiguities in reading off the low energy effective field theory, it is necessary to indicate a neighborhood in moduli space, and in particular more than just a singular Weierstrass model. At a smooth point of moduli space, this involves indicating the value of the complex structure moduli as well as the intermediate Jacobian of the threefold [35-37], and in singular limits, the moduli space is completed by T-brane configurations [36, 37] (see also [38-44]). This is particularly important in the case of $(4,6,12)$ points because motion in a T-brane direction means one is not at a singular point of the effective field theory moduli space. See figure 1 for a depiction of the different deformations of a singular F-theory model.

When the collision of divisors supporting algebras $\mathfrak{g}_{1}$ and $\mathfrak{g}_{2}$ occurs with a sufficiently high order of tangency, additional moduli are also often localized. This in turn leads to the appearance of additional deformations with a weakly coupled interpretation. From a bottom up perspective, a necessary condition for this to occur is that we can match quantities such as the gravitational anomalies of the theory with $6 \mathrm{D}$ conformal matter [4547 with those at the weakly coupled point. From a top down perspective, we can provide checks on our proposal by applying heterotic/F-theory duality in the special limit where the heterotic string propagates on an orbifold. 


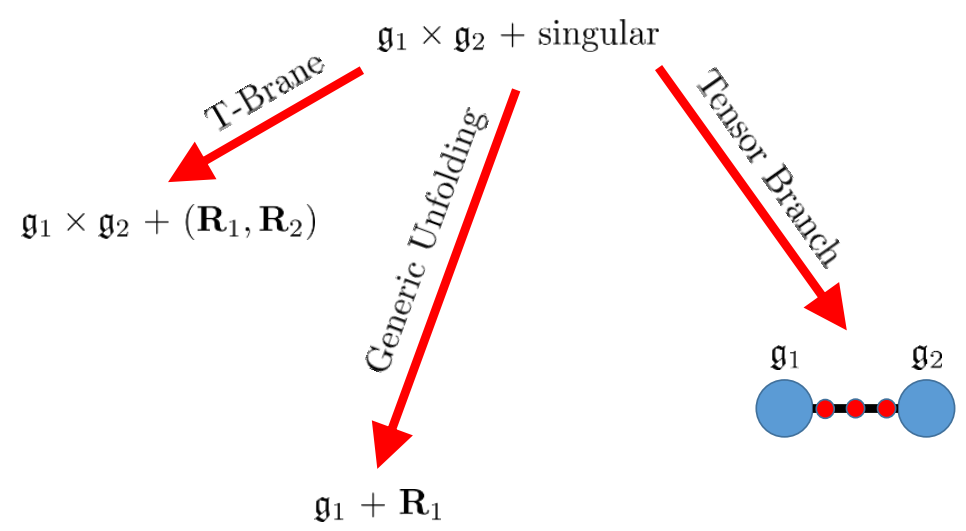

Figure 1. Depiction of the deformations of singular F-theory models which can generate an exotic bifundamental. The minimal Weierstrass model contains points where the multiplicity of vanishing for $(f, g, \Delta)$ is at least $(4,6,12)$. T-brane deformations lead to models with exotic bifundamentals, and tensor branch deformations lead to deformations of 6D SCFTs coupled to gravity.

In this work, we focus on two canonical examples where this phenomenon occurs, though it is clear our method generalizes. First, we consider exotic bifundamental matter in the $(\mathbf{5 6}, \mathbf{2})$ of $\mathfrak{e}_{7} \times \mathfrak{s u}_{2}$. This arises in compactifications of heterotic strings on the orbifold $T^{4} / \mathbb{Z}_{2}$, and the F-theory dual description of this case has been studied for example in [25]. Here, we clarify some aspects of this construction, in particular the structure of the moduli space on the tensor branch of the associated SCFTs. We then turn to exotic bifundamental matter in the $(\mathbf{2 7}, \mathbf{3})$ of $\mathfrak{e}_{6} \times \mathfrak{s u}_{3}$ which arises in compactifications of heterotic strings on the orbifold $T^{4} / \mathbb{Z}_{3}$. In this case, there are additional subtle features in the global F-theory model, since the full gauge algebra is $\mathfrak{e}_{6} \times \mathfrak{s u}_{3} \times \mathfrak{u}(1) \times \mathfrak{e}_{7}$. Both examples have the peculiar feature that a single bifundamental hypermultiplet is spread across several points of the Ftheory base. As such, their appearance cannot be a purely local effect, as it is for standard F-theory matter, and has to be a consequence of the global nature of our models.

The resulting $6 \mathrm{D}$ effective field theories can be further taken as the starting point for realizing additional "exotic structures" in F-theory by further higgsing. Strictly speaking, the compact models we present contain only a single exotic bifundamental, whereas to initiate further breaking patterns it is necessary to have at least two such hypermultiplets. This appears possible to arrange in various local geometries, as well as $4 \mathrm{D}$ models, and suggests a rich class of additional exotic structures.

For example, breaking patterns involving the $(\mathbf{5 6}, \mathbf{2})$ of $\mathfrak{e}_{7} \times \mathfrak{s u}_{2}$ can realize an $\mathfrak{s u}_{2}$ gauge theory with matter in the $\mathbf{5}$ of $\mathfrak{s u}_{2}$, which is higher than the $\mathbf{4}$ of $\mathfrak{s u}_{2}$ realized via matter on a singular divisor (see reference [12]). In the case of the $(\mathbf{2 7}, \mathbf{3})$ of $\mathfrak{e}_{6} \times \mathfrak{s u}_{3}$, a further higgsing yields an $\mathfrak{s u}_{3}$ gauge theory with matter in the three-index symmetric representation (10) of $\mathfrak{s u}_{3}$, thus exceeding the dimension of the two-index symmetric (6) constructed in [26]. Proceeding even further, activating vevs for these high dimension representations leads us to finite non-abelian gauge groups. In the case of $\mathfrak{s u}_{2}$ breaking via a vev for the $\mathbf{5}$ of $\mathfrak{s u}_{2}$ (see, e.g., [48]), this yields the quaternion group, and in the case of $\mathfrak{s u}_{3}$ breaking via vevs for the $\mathbf{1 0}$ as well $\mathbf{6}$ of $\mathfrak{s u}_{3}$ (see, e.g., [49]), this yields $A_{4}$, the alternating group on four letters. 
The rest of this paper is organized as follows. In section 2 we present our proposal for connecting points with "conformal matter singularities" in the presence of higher tangencies of the Weierstrass model with the existence of weakly coupled points in the moduli space. In section 3 we turn to the F-theory realization of the $(\mathbf{5 6}, \mathbf{2})$ of $\mathfrak{e}_{7} \times \mathfrak{s u} \mathbf{u}_{2}$, and in section 4 we analyze the F-theory realization of the $(\mathbf{2 7}, \mathbf{3})$ of $\mathfrak{e}_{6} \times \mathfrak{s u}_{3}$. Section 5 discusses breaking patterns using exotic bifundamentals. We conclude in section 6 and also discuss some future areas of potential investigation. Some additional technical elements are deferred to the appendices.

\section{Prescription for exotic bifundamentals}

In this section we present a general prescription for constructing examples in F-theory of matter in "exotic" bifundamental representations. The main setup we wish to consider involves collisions of two components of the discriminant locus, supporting respective gauge algebras $\mathfrak{g}_{1}$ and $\mathfrak{g}_{2}$. At the collision point, matter will be localized. In terms of the minimal Weierstrass model:

$$
y^{2}=x^{3}+f x+g
$$

the discriminant locus $\Delta=4 f^{3}+27 g^{2}$ factorizes into distinct components:

$$
\Delta=\Delta_{1} \Delta_{2} \cdots
$$

Geometrically, the elliptically fibered Calabi-Yau is ill-defined when the multiplicity of vanishing for $(f, g, \Delta)$ at a point of the base is at least $(4,6,12)$. In this case, blowups of the base are necessary, and the physical interpretation is the appearance of a $6 \mathrm{D}$ superconformal field theory with degrees of freedom localized at this singular point of the geometry $[4,5,29-31]$. When there is a collision but the multiplicity of vanishing is less singular, we instead expect weakly coupled hypermultiplets. For a recent review on the construction of 6D SCFTs in F-theory, see [32].

Following the standard Tate algorithm $[29,50]$, we find that engineering bifundamentals for gauge algebras such as $\mathfrak{e}_{7} \times \mathfrak{s u}_{2}$ or $\mathfrak{e}_{6} \times \mathfrak{s u}_{3}$ inevitably leads to a singular collision point where the elliptic fibers do not remain in Kodaira-Tate form. For example, the collision of a type III* and a type III fiber can, in local coordinates $(t, u)$ of the base, be written as:

$$
y^{2}=x^{3}+t u^{3} x+t^{2} u^{5}
$$

so to interpret this in terms of a Calabi-Yau geometry, we must blow-up the base at the point $t=u=0$. By a similar token, the collision of a type $\mathrm{IV}^{*}$ and a split type IV fiber can be written as:

$$
y^{2}=x^{3}+t^{2} u^{3} x+t^{2} u^{4}
$$

which again suggests the need for a blow-up at the collision point $t=u=0$. The analysis of the resulting F-theory geometries was first carried out in [29, 30], and was subsequently systematized in later work on 6D SCFTs (see [32] for a recent review).

But the physical theory involves additional data beyond that specified by the minimal Weierstrass model. Indeed, in the context of $6 \mathrm{D}$ vacua, the Higgs branch of F-theory vacua 
at a smooth point in moduli space is specified by the complex structure moduli as well as the (Weil) intermediate Jacobian of the Calabi-Yau threefold. In singular limits of complex structure moduli $[30,36,37]$, the moduli of the intermediate Jacobian are interpreted as T-brane data [38-44]. From this perspective, a seemingly singular presentation of the Calabi-Yau geometry may actually reside at a non-singular point of moduli space in the physical theory.

An illustrative example of this kind is the F-theory dual for heterotic strings compactified on a smooth K3 surface with vector bundle specified by the standard embedding, that is, we embed the spin connection in one of the $E_{8}$ factors. The F-theory geometry is the same as the one we would specify for a theory of 24 small $E_{8}$ instantons [30]. We have in both cases (again using local coordinates):

$$
y^{2}=x^{3}+g_{24}(t) u^{5}+f_{8}(t) u^{4} x+g_{12}(t) u^{6}+\ldots
$$

Geometrically, each zero of $g_{24}$ specifies a type II fiber, and the collision with the $E_{8}$ locus (a type $\mathrm{II}^{*}$ fiber) over $u=0$ indicates a superconformal field theory, provided we are at the origin of the moduli space $[4,5]$.

From a physical perspective, we can start at the singular point in moduli space associated with codimension four instantons of the ten-dimensional $E_{8}$ gauge theory, in which case the zeros of $g_{24}$ indicate the locations of these instantons. Dissolving these instantons into flux (by making the instantons have finite size) moves us onto the Higgs branch of the moduli space, and takes us to a non-singular point in moduli space which is smoothly connected to the heterotic tangent bundle description. At this point in moduli space, we have a standard weakly coupled interpretation of the gauge theory and matter content given by an $\mathfrak{e}_{7}$ gauge theory with 20 half-hypermultiplets in the fundamental representation.

We can also coalesce multiple instantons at the same location. For example, the local presentation:

$$
y^{2}=x^{3}+u^{5}\left(u+\alpha t^{k}\right)+\ldots
$$

involves a higher order tangency of intersection at $t=u=0$, and is associated with $k$ small instantons at the same locus. The appearance of more instantons at the same location of the geometry means there are additional moduli are available. Again, in heterotic terms we can dissolve these instantons as flux inside the $E_{8}$ locus, leading to a weakly coupled point of moduli space. In these cases as well, T-brane deformations are available which leave the Weierstrass model unchanged.

Putting these facts together, we see that even when we encounter a singular point where the multiplicity of vanishing for $(f, g, \Delta)$ is at least $(4,6,12)$, it may still be possible to move in Higgs branch moduli space whilst still remaining at a singular point of complex structure moduli. Moreover, we see that by increasing the order of tangency for the collision of components of the discriminant, we have additional moduli. These additional moduli mean there can be further T-brane deformations available to move us to a weakly coupled description. Note that for heterotic strings compactified on a smooth K3 surface, we need at least four small instantons to participate in the breaking pattern to $E_{7}$. The standard way to check whether such a Higgs branch transition is possible is to match various anomalies 
between the tensor branch and Higgs branch descriptions. In particular, we need to ensure that there is no change in the contribution to the gravitational anomaly:

$$
\delta H-\delta V+29 \delta T=0,
$$

where $\delta H$ denotes the change in the number of hypermultiplets, $\delta V$ the change in the number of vector multiplets, and $\delta T$ the change in the number of tensor multiplets.

Taking into account these considerations, we see that even when we encounter collisions of the discriminant locus which do not remain in Kodaira-Tate form, the physical theory may nevertheless be described by a weakly coupled model. From this perspective, to engineer examples of "exotic" bifundamental representations, we apply the following procedure:

- Engineer a collision between two components of the discriminant with gauge algebra $\mathfrak{g}_{1} \times \mathfrak{g}_{2}$.

- Determine the tensor branch by blowing up the collision point, using the algorithm outlined in references $[29,30]$ (see also [31]).

- Passing back to the origin of the tensor branch, increase the order of tangency to generate a higher dimension for the Higgs branch.

- Introduce a candidate weakly coupled gauge theory with bifundamentals with the same gravitational anomaly as the tensor branch theory.

- When available, use a dual heterotic description as a cross check.

In practice, we find that exotic bifundamentals are delocalized across multiple collision points. While it might indeed appear exotic in F-theory, in the hetorotic dual the exotic bifundamental comes from the untwisted sector. This also illustrates that some aspects of exotic bifundamentals are not completely local.

As we have already noted, the step of tuning to increase the order of tangency is sometimes necessary for a T-brane deformation to exist at all. It would be interesting to determine from first principles the minimal order of tangency necessary to accommodate exotic bifundamentals, but in this work we shall confine our analysis to a recursive "trial and error" procedure.

\section{Engineering the $(56,2)$ of $\mathfrak{e}_{7} \times \mathfrak{s u}_{2}$}

In this section we turn to the F-theory realization of $6 \mathrm{D}$ vacua with hypermultiplets transforming in the $(\mathbf{5 6}, \mathbf{2})$ representation of $\mathfrak{e}_{7} \times \mathfrak{s u}_{2}$. As we have already remarked in section 2 , the construction in F-theory is somewhat subtle, in part because such bifundamentals appear to reside at singular points where the elliptic fiber is no longer in Kodaira-Tate form.

On the other hand, we are in some sense guaranteed to find a sensible F-theory realization because there is a well-known way to generate such bifundamental representations via compactifications of the $E_{8} \times E_{8}$ heterotic string on the orbifold geometry $T^{4} / \mathbb{Z}_{2}$. The 
full 6 D theory has gauge algebra $\mathfrak{e}_{7} \times \mathfrak{s u}_{2} \times \mathfrak{e}_{8}$, a single tensor multiplet of charge -12 , and hypermultiplets in the representations

$$
(\mathbf{5 6}, \mathbf{2}, \mathbf{1}) \oplus(\mathbf{5 6}, \mathbf{1}, \mathbf{1})^{\oplus 8} \oplus(\mathbf{1}, \mathbf{2}, \mathbf{1})^{\oplus 32} \oplus(\mathbf{1}, \mathbf{1}, \mathbf{1})^{\oplus 4} .
$$

Since the orbifold $T^{4} / \mathbb{Z}_{2}$ can be viewed as an elliptically fibered $\mathrm{K} 3$ surface with tuned values of the K3 moduli, we expect there to be a corresponding F-theory dual, and therefore, for an F-theory realization to exist. The resulting elliptically fibered Calabi-Yau threefold was identified in [25], but as we shall shortly review, it appears to contain singular collision points which would ordinarily be identified with the presence of a $6 \mathrm{D}$ SCFT. However, by moving in the T-brane branch of moduli space, we can instead pass to a point where we retain a weakly coupled gauge theory description in the low energy effective field theory. This helps to clarify several aspects on the moduli space of these solutions, both in the F-theory and heterotic realizations of this model.

The rest of this section is organized as follows. First, we present some general considerations on the behavior of T-branes in F-theory backgrounds where the heterotic dual geometry is an orbifold. After this, we turn to the study of the moduli space of the F-theory background which contains the $(\mathbf{5 6}, \mathbf{2})$, focussing in particular on the family of elliptically fibered Calabi-Yau threefolds with $\mathbb{F}_{12}$ base [51]. By tuning to a singular point in moduli space, we reach a theory coupled to 6D SCFTs, as well as its Higgs branch and tensor branch deformations. This setting is dual to heterotic compactification on a K3 with 24 mobile instantons, of which the $T^{4} / \mathbb{Z}_{2}$ orbifold is a special limit [52].

\subsection{T-branes and heterotic orbifolds}

To frame the discussion to follow, we now briefly review some qualitative elements of our proposal. Recall that for heterotic strings compactified on an elliptically fibered K3 surface with vector bundle given by the standard embedding (the tangent bundle $T_{\mathrm{K} 3}$ ), the corresponding F-theory dual is described by a singular Weierstrass model with local presentation $[4,5]$ :

$$
y^{2}=x^{3}+g_{24}(s) t^{5}+f_{\mathrm{K} 3} t^{4} x+g_{\mathrm{K} 3} t^{6}+\ldots
$$

with $f_{\mathrm{K} 3}$ and $g_{\mathrm{K} 3}$ the parameters of the minimal Weierstrass model for the dual heterotic K3 geometry. To really specify the geometry, we also need to consider generic points in the complex structure moduli, wherein we work at a non-zero value of the intermediate Jacobian. At the singular point in complex structure moduli, the associated T-brane deformation dissolves the small instantons localized at the zeros of $g_{24}$ to finite size, leading to the heterotic tangent bundle model. Already in this case, we can see that in spite of the singular presentation near various $(4,6,12)$ points, we expect to have a weakly coupled description.

From this starting point of the standard embedding, we can ask what will happen as we further tune the moduli of the $\mathrm{K} 3$ surface so that it degenerates to the orbifold $T^{4} / \mathbb{Z}_{2}$. There are two sorts of tunings we need to arrange to actually generate the standard heterotic orbifold. First, we seek a limit of an elliptically fibered K3 surface which has degenerated to this orbifold (see, e.g., [52]). Second, we must demand that the vector 
bundle used in the standard embedding also degenerates in a suitable fashion to reach this special point in moduli space. In the dual F-theory geometry, we expect to encounter some features such as T-branes, so we should not discard geometries where the multiplicity of vanishing for $(f, g, \Delta)$ is at least $(4,6,12)$. That being said, one way to understand such configurations is to first begin with a generic unfolding, and then perform further tunings in the discriminant locus. With this in mind, we shall first begin with a minimal Weierstrass model where the $\mathfrak{e}_{7}$ is visible in the complex structure:

$$
y^{2}=x^{3}+f_{20}(s) t^{3} x+g_{24}(s) t^{5}+f_{\mathrm{K} 3} t^{4} x+g_{\mathrm{K} 3} t^{6}+\ldots
$$

Further tuning in the parameters $f_{20}, g_{24}, f_{\mathrm{K} 3}, g_{\mathrm{K} 3}$ will then take us to the proposed heterotic dual geometry. An important subtlety in this procedure is that because the heterotic dual has orbifold singularities, we must allow for the possibility that even though we are dealing with a T-brane deformation, the $f_{20}$ deformation parameters are actually "visible" in our presentation. Indeed, from a field theory perspective, the appearance of local $\mathbb{C}^{2} / \mathbb{Z}_{2}$ singularities in the heterotic geometry means that we are dealing with breaking patterns $\mathfrak{e}_{8} \times \mathfrak{s u}_{2} \rightarrow \mathfrak{e}_{7} \times\left(\mathfrak{s u}_{2}\right)_{\text {diag }}$, where the diagonal $\left(\mathfrak{s u}_{2}\right)_{\text {diag }}$ is "visible" in the heterotic and F-theory geometries.

With these preliminaries dealt with, we now turn to the construction of an exotic bifundamental in our geometry.

\subsection{Generic Weierstrass model with $\mathfrak{e}_{7} \times \mathfrak{s} \mathfrak{u}_{2}$}

To set up the notation, note that a Hirzebruch surface $\mathbb{F}_{n}$ is a $\mathbb{P}_{\text {fiber }}^{1}$-fibration over a $\mathbb{P}_{\text {base }}^{1}$. Denoting the homogeneous coordinates along the fiber by $(u, v)$ and those along the base by $(s, t)$, their scaling relations and associated divisor classes are given by the following table:

\begin{tabular}{|l|l|l|l|l|}
\hline & $u$ & $v$ & $s$ & $t$ \\
\hline$[v]$ & 1 & 1 & 0 & 0 \\
{$[s]$} & $n$ & 0 & 1 & 1 \\
\hline
\end{tabular}

The intersection numbers are $[v]^{2}=-n,[s]^{2}=0,[v] \cdot[s]=1$. The geometry also has self-intersection $+n$ curves with divisor class $[u]=[v]+n[s]$. The anti-canonical bundle has class $\bar{K}=[u]+[v]+[s]+[t]=2[v]+(n+2)[s]$. For a Calabi-Yau elliptic fibration over $\mathbb{F}_{n}$, the Weierstrass functions $f$ and $g$ have to have classes $4 \bar{K}$ and $6 \bar{K}$, respectively.

For $\mathbb{F}_{12}$, it is well known that any such elliptic fibration has to have a type $\mathrm{II}^{*}$ fiber over $\{v\}$, which in F-theory corresponds to a "non-higgsable" $\mathfrak{e}_{8}$ gauge factor without any charged matter (see references $[51,53]$ ). To engineer the $\mathfrak{e}_{7} \times \mathfrak{s u}_{2}$ gauge factor, note that in the heterotic spectrum (3.1), there is precisely one bifundamental hypermultiplet. In an Ftheory model with this matter content, anomaly cancellation requires that the intersection number between the $\mathfrak{e}_{7}$ divisor $[\omega]$ and the $\mathfrak{s u}_{2}$ divisor $[\sigma]$ is 12 . Furthermore, the spectrum also dictates that both divisors have genus 0 and self-intersection number 12 . Therefore, a natural choice is that $[\omega]=[\sigma]=[u]$. Exploiting the reparametrization freedom along $\mathbb{P}_{\text {fiber }}^{1}$, we can put the $\mathfrak{e}_{7}$ at $u=0$. This leaves the $\mathfrak{s u}_{2}$ divisor to be a generic +12 curve on 
$\sigma \equiv d_{0} u+p_{12} v=0$, where $d_{0}$ is a constant and $p_{12}$ is a degree 12 homogeneous polynomial in $(s, t){ }^{1}$

The two exceptional groups can be straightforwardly realized by the Weierstrass model:

$$
f=u^{3} v^{4} \tilde{f}, \quad g=u^{5} v^{5} \tilde{g}, \quad \Delta=u^{9} v^{10}(\underbrace{4 v^{2} \tilde{f}^{3}+27 u \tilde{g}^{2}}_{\tilde{\Delta}}) .
$$

The divisor class of the remaining free coefficients are

$$
\begin{aligned}
& {[\tilde{f}]=[u]+8[s]=[v]+20[s],} \\
& {[\tilde{g}]=[u]+[v]+12[s]=2[u]=2[v]+24[s] .}
\end{aligned}
$$

As discussed in [25], this model can be viewed as having higgsed the $\mathfrak{s u}_{2}$ completely with the doublets in (3.1). Importantly, the number of singlets required to cancel the gravitational anomaly agrees with the number of complex parameters in $\tilde{f}$ and $\tilde{g}$ modulo the reparametrization degrees of freedom of $\mathbb{F}_{12}$.

We now employ Tate's algorithm $[50,51]$ to enhance the fiber singularity over $\{\sigma\}$ to $\mathrm{I}_{2}$. Because of the classes (3.6), the most general form of $\tilde{f}$ and $\tilde{g}$ is

$$
\begin{aligned}
\tilde{f}= & \tilde{f}_{20} v+\tilde{f}_{8} \sigma, \\
\tilde{g}= & \tilde{g}_{24} v^{2}+\tilde{g}_{12} v \sigma+\tilde{g}_{0} \sigma^{2} \\
\stackrel{u=\frac{\sigma-p v}{d_{0}}}{\Longrightarrow} \tilde{\Delta}= & \frac{v^{5}}{d_{0}}(\underbrace{4 d_{0} \tilde{f}_{20}^{3}-27 \tilde{g}_{24}^{2} p_{12}}_{\tilde{\Delta}_{0}}) \\
& +\frac{3 v^{4}}{d_{0}} \underbrace{4 d_{0} \tilde{f}_{20}^{2} \tilde{f}_{8}+9 \tilde{g}_{24}\left(\tilde{g}_{24}-2 \tilde{g}_{12} p_{12}\right)}_{\tilde{\Delta}_{1}}) \sigma+\mathcal{O}\left(\sigma^{2}\right) .
\end{aligned}
$$

Note that $\tilde{g}_{0}$ is not really an independent free parameter, because it can be absorbed in $\sigma$ by the coefficient $d_{0}$. To obtain an $\mathfrak{s u}_{2}$ on $\sigma=0$, we have to tune $\tilde{\Delta}_{0}=\tilde{\Delta}_{1}=0$, keeping in mind that these are polynomials over $\mathbb{P}_{\text {base }}^{1}$ and thus elements of a unique factorization domain (UFD). Explicitly, requiring $\tilde{\Delta}_{0}=4 d_{0} \tilde{f}_{20}^{3}-27 \tilde{g}_{24}^{2} p_{12}=0$ means that prime factors of $\tilde{f}_{20}$ have to contain those of $p_{12}$. However, because of the cube, $\tilde{g}_{24}$ must then also contain a factor of $p_{12}$, i.e.,

$$
\tilde{f}_{20}=c_{8} p_{12}, \quad \tilde{g}_{24}=c_{12} p_{12} \quad \Longrightarrow \quad \tilde{\Delta}_{0}=p_{12}^{3}\left(4 d_{0} c_{8}^{3}-27 c_{12}^{2}\right) .
$$

To set this to zero, we therefore need $c_{8} \sim c_{4}^{2}$ and $c_{12} \sim c_{4}^{3}$ for an arbitrary degree 4 polynomial $c_{4}$. The coefficients turn out to be such that

$$
\tilde{f}_{20}=\frac{1}{48} p_{12} c_{4}^{2}, \quad \tilde{g}_{24}=\frac{\sqrt{d_{0}}}{864} p_{12} c_{4}^{3},
$$

which, as can be verified, sets $\tilde{\Delta}_{0}=0$. Substituting into $\tilde{\Delta}_{1}$ yields

$$
\tilde{\Delta}_{1} \sim \sqrt{d_{0}}\left(c_{4}^{2}+144 \tilde{f}_{8}\right) c_{4}-1728 \tilde{g}_{12} \stackrel{!}{=} 0,
$$

\footnotetext{
${ }^{1}$ The constant $d_{0}$ could also be absorbed into $p_{12}$, so that $\sigma=u+p_{12} v$. However, we keep it here in order to have the same notation as in [25].
} 
which requires $\tilde{g}_{12}=\frac{\sqrt{d_{0}}}{1728} c_{4}\left(c_{4}^{2}+144 \tilde{f}_{8}\right)$. Making the redefinitions $c_{4}=12 d_{4} / \sqrt{d_{0}}, c_{8}=$ $d_{8} / d_{0}, g_{0}=1 / d_{0}$ (recall that $g_{0}$ was not independent of $d_{0}$ ), the result of the above tuning is the Weierstrass model

$$
\begin{aligned}
& f=\frac{u^{3} v^{4}}{d_{0}}\left(d_{0} d_{8} u+\left(3 d_{4}^{2}+d_{8}\right) p_{12} v\right) \\
& g=\frac{u^{5} v^{5}}{d_{0}}\left(d_{0}^{2} u^{2}+d_{0}\left(d_{4}^{3}+d_{4} d_{8}+2 p_{12}\right) u v+\left(3 d_{4}^{3}+d_{4} d_{8}+p_{12}\right) p_{12} v^{2}\right), \\
& \Delta=\frac{u^{9} v^{10}}{d_{0}^{3}}\left(d_{0} u+p_{12} v\right)^{2} \underbrace{\left(27 d_{0}^{3} u^{3}+(\ldots) u^{2} v+(\ldots) u v^{2}+4\left(3 d_{4}^{2}+d_{8}\right)^{3} p_{12} v^{3}\right)}_{\Delta_{\mathrm{res}}} .
\end{aligned}
$$

The unspecified parameters are in the polynomials of the indicated degrees, $d_{0}, d_{4}, d_{8}, p_{12}$, which yields a total of 28 . Of these, 3 can be removed by the $\mathrm{SL}(2, \mathbb{C})$ reparametrization freedom on $\mathbb{P}_{\text {base }}^{1}$. By insisting on the $\mathfrak{e}_{7}$ and $\mathfrak{e}_{8}$ divisors be on $u=0$ and $v=0$, there is only the rescaling freedom left on the fiber $\mathbb{P}^{1}$, which removes another free parameter. Thus, there are $28-4=24$ independent deformation parameters preserving the $\mathfrak{e}_{7} \times \mathfrak{s u}_{2}$ gauge group, i.e., there are 24 neutral hypermultiplets.

Charged hypermultiplets are localized at intersections of discriminant components. At the intersection $u=p_{12}=0$ of the $\mathfrak{s u}_{2}$ and $\mathfrak{e}_{7}$ divisors, the vanishing orders of $(f, g, \Delta) \sim(4,6,12)$ indicate non-minimal singularities and will require further discussion. To analyze other matter loci, we take a closer look at the $\mathrm{I}_{1}$ locus over the residual discriminant component $\Delta_{\text {res }}=0$. First, the only enhancement points on the $\mathfrak{e}_{7}$ divisor other than the non-minimal points are at $3 d_{4}^{2}+d_{8}=0$. Importantly, the coefficient of $u v^{2}$ does not factor this polynomial, which also implies that the vanishing orders of the Weierstrass functions (3.11) at $u=0=3 d_{4}^{2}+d_{8}$ are $(f, g, \Delta) \sim(4,5,10)$, indicating the presence of $\frac{1}{2} \mathbf{5 6}$ 's. Since $3 d_{4}^{2}+d_{8}$ has degree 8 , there are therefore 8 half-hypermultiplets of $(\mathbf{5 6}, \mathbf{1})$ matter.

For the enhancement points on the $\mathfrak{s u}_{2}$ divisor $\sigma=d_{0} u+p_{12} v$, we rewrite the residual discriminant as

$$
\Delta_{\mathrm{res}}=27 \sigma^{3}+(\ldots) \sigma^{2} v+(\ldots) \sigma v^{2}+Q_{16} p_{12} d_{4}^{2} v^{3},
$$

where neither the ellipses in parentheses nor the degree 16 polynomial $Q_{16}$ contain any overall factor of $d_{4}$ or $\sigma$. Besides the non-minimal points at $p_{12}=0$, there are two other types of enhancements. By simply checking the vanishing orders of the Weierstrass functions, we identify enhancements to $I_{3}$ resp. type III at $Q_{16}=0$ resp. $d_{4}=0$. The enhancement to $\mathrm{I}_{3}$ occur at normal crossings with the $\mathrm{I}_{1}$ component at $\sigma=Q_{16}=0$ and thus lead to 16 hypermultiplets of $(\mathbf{1}, \mathbf{2})$. On the other hand, the type III enhancement points with no matter are at order 2 tangency points $\sigma=d_{4}=0$, where the residual discriminant goes like $\Delta_{\text {res }} \approx \sigma+d_{4}^{2}$.

Finally, the non-minimal points at $u=p_{12}=0$ are transverse intersections between the $\mathfrak{e}_{7}, \mathfrak{s u}_{2}$ divisors and the residual discriminant. To resolve these, we have to blow-up the base with twelve exceptional divisors at each root of $p_{12}=u=0$. This introduces twelve 
$(-1)$-curves in the base, over which the elliptic fiber is easily checked to be smooth and thus no further matter states at their intersections with $\{u\}$ and $\{\sigma\}$. Because all twelve blow-ups occur on the $\mathfrak{e}_{7}$ and $\mathfrak{s u}_{2}$ divisors, each of their self-intersection number is lowered by 12 , thus we end with self-intersection 0 curves in both cases. This is consistent with anomaly cancellation, which requires precisely 4, resp. 16 fundamental hypermultiplets. The twelve blow-ups further introduce twelve tensor multiplets, which contribute to the gravitational anomaly. Taking into account the spectator $\mathfrak{e}_{8}$, we thus have:

$$
\begin{aligned}
T & =13, \\
V & =248+133+3=384, \\
H_{\text {charged }} & =4 \cdot 56+16 \cdot 2=256, \\
H_{\text {neutral }} & =24 \\
\Longrightarrow \quad H-V+29 T & =273 .
\end{aligned}
$$

\subsection{Orbifold conditions from heterotic/F-theory duality}

So far, we have not paid attention to the heterotic dual of this F-theory model on $\mathbb{F}_{12}$. By heterotic/F-theory duality, the heterotic compactification space is a K3 surface inside the F-theory threefold that is elliptically fibered over $\mathbb{P}_{\text {base }}^{1}$. To reach the $T^{4} / \mathbb{Z}_{2}$ orbifold point in moduli space, the heterotic K3 first has to be tuned such that its elliptic fibration has four $\mathrm{I}_{0}^{*}$ fibers. Each such fiber contributes four volume moduli, which together with the volume of the base and the generic fiber yield 18 Kähler parameters, leaving two complex structure parameters inside $h^{1,1}(\mathrm{~K} 3)=20$. Of these, 16 of the Kähler moduli have to be fixed to shrink all but the middle component of the $\mathrm{I}_{0}^{*}$ fibers to zero size. The four remaining free parameters (two complex and two Kähler) form the four singlets in the heterotic spectrum (3.1).

As pointed out in [25], one should identify the heterotic K3 inside the F-theory model by expanding the F-theory Weierstrass functions $(f, g)$ in the variables $(u, v)$,

$$
f=\sum_{k \geq 0} f_{56-12 k} u^{k} v^{8-k}, \quad g=\sum_{k \geq 0} g_{84-12 k} u^{k} v^{12-k},
$$

where the coefficients are homogeneous polynomials over $\mathbb{F}_{12}$ 's base $\mathbb{P}^{1}$ of degree given by the subscript. The elliptic fibration structure of the K3 is then described by the Weierstrass model $\left(f_{\mathrm{K} 3}, g_{\mathrm{K} 3}\right)=\left(f_{8}, g_{12}\right)$. Furthermore, the 24 mobile instantons of the heterotic theory are localized in the fibers of the K3 over the zeroes of $g_{24}$. For the heterotic K3 to have $\mathrm{I}_{0}^{*}$ fibers at four points, which we can without loss of generality assume to be the roots of a non-degenerate degree four polynomial $p_{4}$ over $\mathbb{P}_{\text {base }}^{1}$, we need to tune $f_{8} \sim p_{4}^{2}$ and $g_{12} \sim p_{4}^{3}$. Additionally, the mobile instantons have to be trapped at the fixed points in the orbifold limit. These fixed points correspond to the singularities of the K3 which are in the $\mathrm{I}_{0}^{*}$ fibers, hence we also need $g_{24} \sim p_{4}^{6}$.

Applying these criteria to the generic Weierstrass model (3.11) with $\mathfrak{e}_{7} \times \mathfrak{s u}_{2}$, we obtain the conditions

$$
\tilde{f}=d_{0} d_{8} \sim p_{4}^{2}, \quad \tilde{g}=d_{0}\left(d_{4}^{3}+d_{4} d_{8}+2 p_{12}\right) \sim p_{4}^{3}, \quad g_{24}=\left(3 d_{4}^{3}+d_{4} d_{8}+p_{12}\right) p_{12} \sim p_{4}^{6},
$$


The minimal (i.e., removing the least number of free parameters) tuning necessary is

$$
d_{4}=\beta p_{4}, \quad d_{8}=\alpha p_{4}^{2}, \quad p_{12}=p_{4}^{3},
$$

for some complex numbers $\alpha, \beta$. The resulting elliptic fibration describes the F-theory geometry dual to the $T^{4} / \mathbb{Z}_{2}$ orbifold [25]:

$$
\begin{aligned}
& f=\frac{p_{4}^{2}}{d_{0}} u^{3} v^{4}\left(d_{0} u+\left(\alpha+3 \beta^{2}\right) p_{4}^{3} v\right) \\
& g=\frac{1}{d_{0}} u^{5} v^{5}\left(d_{0}^{2} u^{2}+d_{0}\left(2+\alpha \beta+\beta^{3}\right) p_{4}^{3} u v+\left(1+\alpha \beta+3 \beta^{3}\right) p_{4}^{6} v^{2}\right) \\
& \Delta=\frac{1}{d_{0}^{3}} \underbrace{u^{9}}_{E_{7}} \underbrace{v^{10}}_{E_{8}}(\underbrace{d_{0} u+p_{4}^{3} v}_{\mathrm{SU}(2)})^{2}(\underbrace{\lambda_{1} u^{3}+\lambda_{2} p_{4}^{3} u^{2} v+\lambda_{3} p_{4}^{6} u v^{2}+\lambda_{4} p_{4}^{9} v^{3}}_{\Delta_{\text {res }}}) \\
& \lambda_{1}=27 d_{0}^{3}, \quad \lambda_{2}=54 d_{0}^{2}\left(1+\alpha \beta+\beta^{3}\right), \quad \lambda_{4}=4\left(\alpha+3 \beta^{2}\right)^{3}, \\
& \lambda_{3}=d_{0}\left(27+4 \alpha^{3}+54 \alpha \beta+27 \alpha^{2} \beta^{2}+162 \beta^{3}+54 \alpha \beta^{4}+27 \beta^{6}\right) .
\end{aligned}
$$

with

This Weierstrass model has eight free parameters in terms of $p_{4}, d_{0}, \alpha, \beta$. Subtracting the $3+1$ reparametrization choices left on $\mathbb{F}_{12}$, it leaves precisely 4 singlets, as required by the perturbative heterotic spectrum (3.1).

By inspection of the minimal Weierstrass model, we see various non-minimal points with order of vanishing for $(f, g, \Delta)$ at least $(4,6,12)$. In this respect, our analysis supplements the considerations of [25] because we have identified the appearance of T-brane deformations, which move us away from the singular point in moduli space. At such a point, blowing up the base is not physically allowed. This is in accord with the considerations of reference [25]. Of course, we can also move back to the singular point of the Higgs branch moduli space, and then move onto the tensor branch. We turn to an analysis of this next.

\subsection{The strongly coupled sector of the global model}

The above tuning process was solely justified through the duality to heterotic theory. In the following, we will discuss the model from a purely F-theory perspective and elucidate the connection to strongly coupled physics.

One key feature of the tuning process is that it changes the type of intersection between the $\mathfrak{e}_{7}$ divisor with the other discriminant components from transverse to tangential with order three. From an F-theory perspective, this seems to be a necessary condition for the appearance of matter in the $\mathbf{5 6}$ of $\mathfrak{e}_{7}$. Indeed, the standard Weierstrass model with $\mathfrak{e}_{7}$ on a divisor $\{w\}, y^{2}=x^{3}+w^{3} \tilde{f} x+w^{5} \tilde{g}$, has a residual discriminant of the form $\tilde{\Delta}=27 \tilde{g}^{2} w+4 \tilde{f}^{3}$. For generic $\tilde{g}$, the residual discriminant at $w=\tilde{f}=0$ is locally described by the curve $w \sim \tilde{f}^{3}$, indicating that the 7-brane along $\tilde{\Delta}=0$ is intersecting the $\mathfrak{e}_{7}$ stack at $w=0$ with an order three tangency. In the generic case, the singularity of the fiber enhances to $\mathfrak{e}_{8}$, which according to the Katz-Vafa rules [29, 54], gives rise to a hypermultiplet of $\frac{1}{2} \mathbf{5 6}$.

From this perspective, it is expected that the "generic" Weierstrass model (3.11) with $\mathfrak{e}_{7} \times \mathfrak{s u}_{2}$ gauge group cannot support matter in the $\mathbf{5 6}$ at the intersection between the 
divisors. In that case, the residual discriminant factors into two components. While the $\mathrm{I}_{1}$ part still intersects the $\mathfrak{e}_{7}$ divisor $\{u\}$ tangentially at $u=3 d_{4}^{2}+d_{8}=0$, giving rise to 56's, the $\mathrm{I}_{2}$ component along $\left\{d_{0} u+p_{12} v\right\}$ now intersects $\{u\}$ transversely at $u=p_{12}=0$. Note that the global nature of the model forces the $\mathrm{I}_{1}$ to also intersect $\{u\}$ transversely at this point, preserving the multiplicity three of the total intersection at each meeting point between the $\mathfrak{e}_{7}$ and the rest of the discriminant. The resulting non-minimal singularity can only be resolved by blowing up the base.

However, the tangency argument alone is not enough to justify the tuning (3.16) that the heterotic dual requires. This can be easily seen as it would have sufficed to set $p_{12}=$ $p_{4}^{3}$ in (3.11), which would have made the $\mathfrak{e}_{7}$ divisor intersect the $\mathrm{I}_{2}$ and $\mathrm{I}_{1}$ discriminant components tangential with order three. So how can we make sense of the additional tunings $d_{4} \sim p_{4}, d_{8} \sim p_{4}^{2}$ that are required to reach the orbifold limit in F-theory? To shed some light on this issue, it proves to be instructive to again resolve the non-minimal points by blow-up, i.e., we go onto the tensor branch of the theory. It is straightforward to check that the partial tuning $p_{12}=p_{4}^{3}$ does not change the gauge sector on the tensor branch: ${ }^{2}$ Though we have grouped the 12 non-minimal points into four groups of three, the vanishing order of $(f, g, \Delta)$ is still $(4,6,12)$. These points are still resolved with 12 exceptional curves, now three at each point, over all of which the elliptic fiber remains smooth. Therefore, the numbers of tensors, hypers and vectors remain as in (3.13) in order to cancel the gravitational anomaly, i.e., we have not really lost the singlets by going from $p_{12}$ to $p_{4}^{3}$.

The situation changes considerably when we include also the other two tuning conditions. By setting $d_{4} \sim p_{4}, d_{8} \sim p_{4}^{2}$, all the codimension two enhancement loci - those supporting the $(\mathbf{5 6}, \mathbf{1})$ and $(\mathbf{1}, \mathbf{2})$ matter states as well as the type III fibers on the $\mathfrak{s u}_{2}$ locus - are collapsed onto the non-minimal point at $u=p_{4}=0$. The vanishing orders are now enhanced to $(f, g, \Delta) \sim(6,7,14)$. By again blowing up these points in the base, we now observe that the tensor branch theory has changed drastically: now we need five blow-ups at each point to resolve the singularity, and these curves are decorated with singular Kodaira fibers over them. The situation is summarized in figure 2. The result of this blow-up process is a new gauge sector at each previously non-minimal point, consisting of the gauge factors $\mathfrak{s u}_{2} \times \mathfrak{g}_{2} \times \mathfrak{s u}_{2}$ with matter $\frac{1}{2}[(\mathbf{2}, \mathbf{1}, \mathbf{1}) \oplus(\mathbf{2}, \mathbf{7}, \mathbf{1}) \oplus(\mathbf{1}, \mathbf{7} \oplus \mathbf{1}, \mathbf{2})] \oplus(\mathbf{1}, \mathbf{7}, \mathbf{1})$. In addition, the $\mathfrak{s u}_{2}$ divisor $\{\sigma\}$ passes through all four $\mathfrak{g}_{2}$ divisors, with each intersection contributing a $\frac{1}{2}(\mathbf{7} \oplus \mathbf{1}, \mathbf{2})$ hypermultiplet. One can check that the gauge anomalies of each of the blow-up sectors is cancelled with this spectrum (all gauge groups introduced by blow-ups are on -2 curves). Through the blow-up process, the self-intersection of the $\mathfrak{e}_{7}$ divisor has decreased to $12-4 \cdot 5=-8$, which is required by the anomaly conditions for no charged matter. The self-intersection of the $\mathrm{I}_{2}$ divisor on $\sigma$ changes to $12-4 \cdot 3=0$ - the first three blow-ups are enough to remove any non-minimal points on it (see figure 2) which is also consistent with having in total $4 \times \frac{1}{2} \times 8=16$ fundamental hypermultiplets.

\footnotetext{
${ }^{2}$ The charge lattice of strings does change, however.
} 

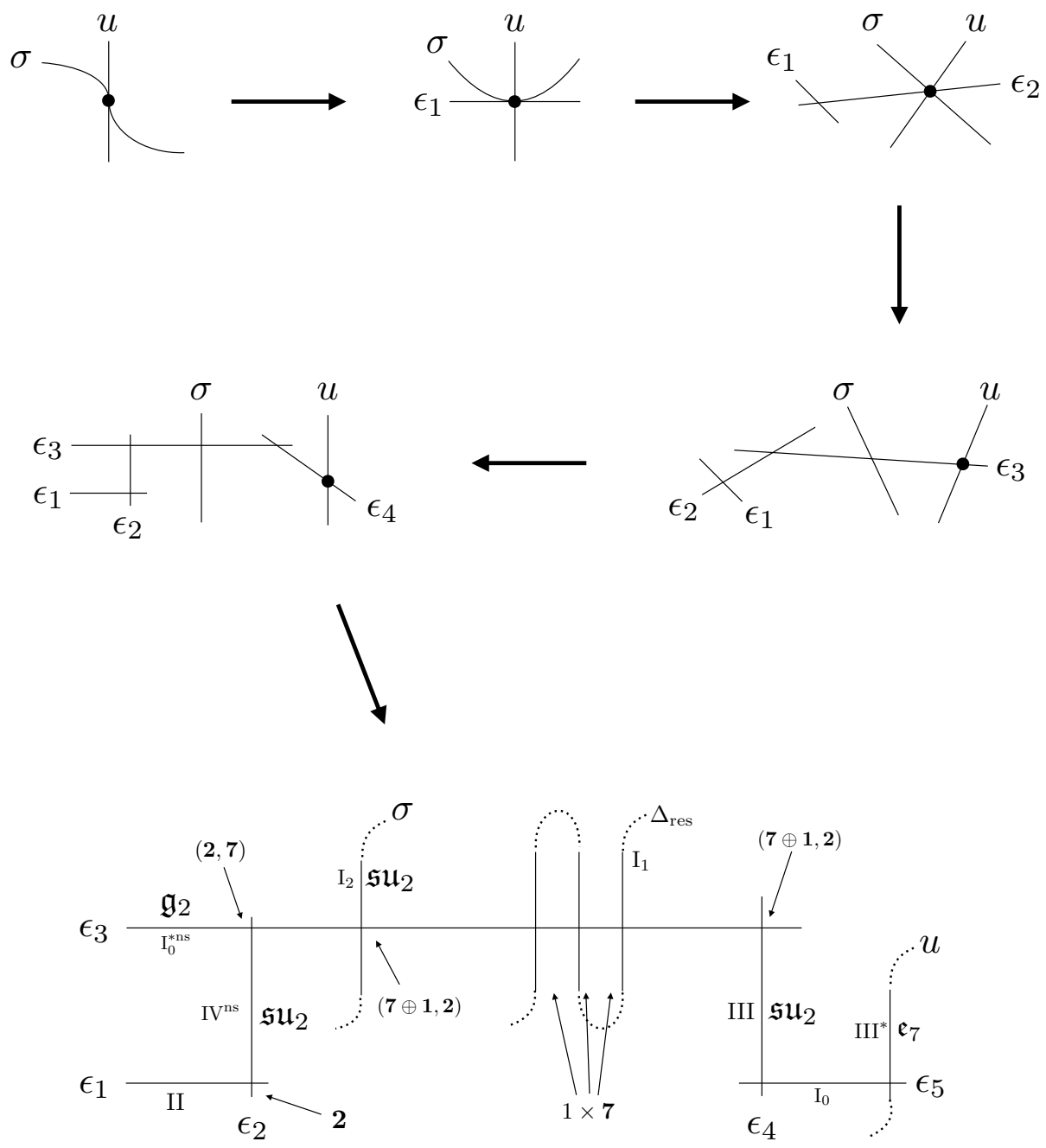

Figure 2. Blow-up of the base at one of the four points $u=p_{4}=0$, where the $\mathfrak{e}_{7}$ divisor $\{u\}$ and the $\mathfrak{s u}_{2}$ divisor $\left\{\sigma \equiv d_{0} u+p_{4}^{3} v\right\}$ intersect. It requires five $\mathbb{P}^{1}$ 's $\left\{\epsilon_{i}\right\}$, carrying the indicated Kodaira fibers and associated gauge algebras, to resolve all non-minimal points (black dots). After the blow-ups the curve $\{u\}$ has self-intersection -8 , and $\{\sigma\}$ has 0 . All of the exceptional curves have self-intersection -2 , except for $\left\{\epsilon_{5}\right\}$ which has -1 . All displayed matter multiplets are halfhypermultiplets, except the $\mathbf{7}$ of $\mathfrak{g}_{2}$ on $\left\{\epsilon_{3}\right\}$, which is a full hypermultiplet spread across the three intersection points with the residual discriminant.

The full spectrum on the tensor branch is thus

$$
\begin{aligned}
H_{\text {charged }} & =4 \times \#\left(\frac{1}{2}[(\mathbf{2}, \mathbf{1}, \mathbf{1}) \oplus(\mathbf{2}, \mathbf{7}, \mathbf{1}) \oplus(\mathbf{1}, \mathbf{7} \oplus \mathbf{1}, \mathbf{2})] \oplus(\mathbf{1}, \mathbf{7}, \mathbf{1}) \oplus \frac{1}{2}(\mathbf{7} \oplus \mathbf{1}, \mathbf{2})\right) \\
& =124 \\
V & =\operatorname{dim}\left(\mathfrak{e}_{7} \times \mathfrak{s u}_{2} \times \mathfrak{e}_{8} \times\left(\mathfrak{s u}_{2} \times \mathfrak{g}_{2} \times \mathfrak{s u}_{2}\right)^{\otimes 4}\right)=464, \\
T & =1+4 \cdot 5=21 \\
\Longrightarrow 273 & =H_{\text {charged }}-V+29 T+4,
\end{aligned}
$$

which confirms that there are only four singlets in the theory. 
The strongly coupled sector generated in this way is also interesting in its own right. Taking a decompactification limit for the curves supporting the $\mathfrak{e}_{7}$ and $\mathfrak{s u}_{2}$ gauge algebra factors, we are left with the tensor branch of a 6D SCFT (actually four copies) with tensor branch description (with notation as in reference [31, 45, 47] as well as the review [32]):

$$
\left[E_{7}\right], 1, \stackrel{\mathfrak{s u}_{2}}{2}, \underset{\left[S p_{2}\right]}{\stackrel{\mathfrak{g}_{2}}{2}}, \stackrel{\mathfrak{s u}_{2}}{2}, 2
$$

In this SCFT there is an emergent $S p_{2} \simeq \operatorname{Spin}_{5}$ flavor symmetry. Indeed, we have a half hypermultiplet in the representation $(\mathbf{7}, \mathbf{4})$ of $\mathfrak{g}_{2} \times \mathfrak{s p}_{2}$. The $\mathfrak{s p}_{2}$ flavor symmetry has a maximal $\left(\mathfrak{s u}_{2}\right)_{L} \times\left(\mathfrak{s u}_{2}\right)_{R}$ subalgebra, one factor of which is identified with the gauge algebra of the compact model. From this perspective, our discussion also provides additional examples of 6D SCFTs coupled to gravity. See e.g. [10, 33, 34] for some earlier examples. Note also that in the limit where we decouple gravity (and the $E_{7}$ and $S p_{2}$ are flavor symmetries) and proceed back to the SCFT point, the $(\mathbf{5 6}, \mathbf{2})$ is a decoupled free hypermultiplet which is delocalized across the four collision points of the $\mathfrak{e}_{7}$ and $\mathfrak{s u}_{2}$ loci. Such scenarios, where single hypermultiplets are spread across several enhancement points, are not uncommon; in fact we have just seen above a similar fate for the $\mathbf{7}$-representation of $\mathfrak{g}_{2}$ in the strongly coupled sector over three points. However, such a delocalization of matter states is usually associated with matter charged under non-simply laced algebras, where we have the well-understood effect of Tate-monodromy. For the gauge algebra $\mathfrak{e}_{7} \times \mathfrak{s u}_{2}$, there is no known geometric mechanism which spreads one hypermultiplet across four codimension two loci. This further emphasizes the importance of the global construction, as well as the non-local nature of the relevant T-brane deformation, which emerges only when we gauge the diagonal subalgebra in the flavor symmetry of four strongly coupled sectors of type (3.19).

With this discussion in hand, we can summarize the significance of the tuning (3.16) as enhancing the strongly coupled sector, which is always accessible via the tensor branch. However, this highly tuned and singular F-theory model is anomaly equivalent to the heterotic orbifold with a perturbative spectrum (3.1). This is a strong indication that at the origin of the tensor branch of the tuned model (3.17) the strongly coupled sector has a Higgs branch, on which the heterotic orbifold model with the perturbative spectrum (3.1) sits. In reference [25], a matching of the spectrum was provided by performing various complex structure deformations, and then tuning back to the singular point in complex structure moduli. From this perspective, we can instead consider deformations involving the intermediate Jacobian. In this case, the general description provided in [36] (see also [37]) indicates that in the limit where the complex structure moduli are tuned to a singular value, we can remain in a weakly coupled regime due to the presence of a T-brane deformation.

\subsection{Adding more bifundamentals}

The presentation given thus far has relied on the existence of a globally complete heterotic dual. In this case, we observe that we can manage to get a single bifundamental, which is delocalized across four points of the geometry. It is natural to ask whether we can modify this construction to produce examples with more than one bifundamental. 
Considering that our criterion is primarily local in nature, we can in principle relax any constraints on the self-intersection numbers of the curves supporting the $\mathfrak{e}_{7}$ and $\mathfrak{s u}_{2}$ locus to produce local geometries with these gauge symmetries and a bifundamental between them. Of course, it will be more challenging to construct a fully compact model, and given the fact that the curves supporting our gauge divisors have positive self-intersection number, there is a sense in which $6 \mathrm{D}$ supergravity must always be reintroduced.

Focussing on the purely gauge theoretic aspects of $6 \mathrm{D}$ anomaly cancellation, the relevant constraints are (see e.g. $[51,55])$ :

$$
\begin{aligned}
\left(\frac{D_{\mathfrak{e} 7} \cdot D_{\mathfrak{e} 7}}{2}+4\right) & =N_{(\mathbf{5 6}, \mathbf{1})}+2 \times N_{(\mathbf{5 6}, \mathbf{2})} \\
\left(6 D_{\mathfrak{s u}(2)} \cdot D_{\mathfrak{s u}(2)}+16\right) & =N_{(\mathbf{1}, \mathbf{2})}+56 \times N_{(\mathbf{5 6}, \mathbf{2})} \\
D_{\mathfrak{e}_{7}} \cdot D_{\mathfrak{s u}(2)} & =12 \times N_{(\mathbf{5 6}, \mathbf{2})},
\end{aligned}
$$

where here, $D_{\mathfrak{g}}$ denotes the divisor class of a curve wrapped by a seven-brane with gauge algebra $\mathfrak{g}$, and the $N$ 's indicate the multiplicity of various representations.

Increasing the number of bifundamentals necessarily raises the self-intersection number relative to the case of a single bifundamental. To see why, consider the extreme example where all matter comes from a bifundamental. This can be satisfied if the divisors supporting gauge algebras intersect as:

$$
\begin{aligned}
& N_{(\mathbf{5 6}, \mathbf{2})}=2, \quad N_{(\mathbf{5 6}, \mathbf{1})}=0, \quad N_{(\mathbf{1}, \mathbf{2})}=0, \\
& D_{\mathfrak{e} 7} \cdot D_{\mathfrak{e} 7}=0, \quad D_{\mathfrak{s u}(2)} \cdot D_{\mathfrak{s u}(2)}=16, \quad D_{\mathfrak{e} 7} \cdot D_{\mathfrak{s u}(2)}=24 .
\end{aligned}
$$

At this level of analysis, there are many examples of this sort. Clearly, gravitational anomalies will impose additional conditions, but if we permit ourselves to consider arbitrary bases, it is a priori possible that all such anomaly cancellation conditions can be satisfied. We leave a full analysis of such examples for future work.

We can also descend to $4 \mathrm{D}$ vacua by compactifying on a matter curve, and in such models the constraints on the number of exotic bifundamentals are likely substantially weaker. The construction of a Calabi-Yau fourfold with the requisite singularities will of course be highly tuned, and will exhibit singularities along curves (see e.g. [56]), and possibly at various Yukawa points as well [57]. Restricting our attention to a purely field theory problem decoupled from gravity, we note that compactification of a $6 \mathrm{D}$ hypermultiplet on a genus $g$ curve $\Sigma$ yields, in the absence of any fluxes, $h^{0}\left(K_{\Sigma}^{1 / 2}\right)=g 4 \mathrm{D} \mathcal{N}=1$ chiral multiplets in the bifundamental representation $(\mathbf{5 6 , 2})$, and $h^{1}\left(K_{\Sigma}^{1 / 2}\right)=h^{0}\left(K_{\Sigma}^{1 / 2}\right)=g 4 \mathrm{D} \mathcal{N}=1$ chiral multiplets in the conjugate representation. From this perspective, it is natural to expect there to be many ways to generate $4 \mathrm{D}$ vacua with a large number of exotic bifundamentals.

\section{Engineering the $(27,3)$ of $\mathfrak{e}_{6} \times \mathfrak{s u _ { 3 }}$}

As a second example, we construct an F-theory model that has bifundamental matter under $\mathfrak{e}_{6} \times \mathfrak{s u}_{3}$. For that, we again rely on heterotic/F-theory duality to have a reference model. 
Indeed, there is a heterotic $\mathbb{Z}_{3}$-orbifold which contains the desired gauge symmetry and matter. Labeled as model IIId in [24], the gauge algebra is $\mathfrak{e}_{6} \times \mathfrak{s u}_{3} \times \mathfrak{e}_{7} \times \mathfrak{u}(1)$, with the following spectrum: ${ }^{3}$

$$
\begin{aligned}
(\mathbf{2 7}, \mathbf{3}, \mathbf{1})_{0} \oplus(\mathbf{2 7}, \mathbf{1}, \mathbf{1})_{\frac{1}{3}}^{\oplus 9} \oplus & (\mathbf{1}, \mathbf{3}, \mathbf{1})_{\frac{2}{3}}^{\oplus 9} \oplus(\mathbf{1}, \mathbf{3}, \mathbf{1})_{-\frac{1}{3}}^{\oplus 18} \\
& \oplus(\mathbf{1}, \mathbf{1}, \mathbf{5 6})_{\frac{1}{2}} \oplus(\mathbf{1}, \mathbf{1}, \mathbf{1})_{1} \oplus(\mathbf{1}, \mathbf{1}, \mathbf{1})_{0}^{\oplus 2} .
\end{aligned}
$$

Cancelling the resulting gauge anomalies in F-theory requires the self- and mutual intersection number of both the $\mathfrak{e}_{6}$ and $\mathfrak{s u}_{3}$ divisors to be 6 , as well as the self-intersection number of the $\mathfrak{e}_{7}$ divisor to be -6 . Thus, the natural choice for the F-theory base is $\mathbb{F}_{6}$, with $\mathfrak{e}_{7}$ on the unique -6-curve $\{v\}$ and the $\mathfrak{e}_{6}$ and $\mathfrak{s u}_{3}$ divisors on +6 -curves $\{u\}$ and $\left\{\sigma \equiv u+p_{6}\right\}$, respectively, with $p_{6}$ a homogeneous degree six polynomial on the $\mathbb{P}_{\text {base }}^{1}$ of $\mathbb{F}_{6}$.

Following the extensive discussion in the $T^{4} / \mathbb{Z}_{2}$ case, we will use the two criteria developed in section 3.4, namely the correct order of tangential intersections and the enhancement of the tensor branch theory, to construct the F-theory dual of the $\mathbb{Z}_{3}$-orbifold. However, due to the global nature of $\mathfrak{u}(1)$ 's in F-theory, our local criteria cannot fully determine the necessary tuning. Physically, the missing tuning conditions are reflected in a miscount of the singlets, which can be especially delicate in an interplay with a $\mathfrak{u}(1)$.

In order to have a good starting point, we begin with a model over $\mathbb{F}_{6}$ having $\mathfrak{e}_{6} \times \mathfrak{s u} \mathfrak{u}_{3} \times \mathfrak{e}_{6}$ constructed via Tate's algorithm, as detailed in appendix A. Here, the second $\mathfrak{e}_{6}$ is the non-higgsable algebra on the -6 curve $\{v\}$. In order to enhance to $\mathfrak{e}_{7}$, as required for the heterotic orbifold, we simply set the parameter $g_{0}=0$. The resulting model

$$
\begin{aligned}
f= & {\left[f_{2} u^{2}+\left(2 f_{2} p_{6}-3 c_{2}^{4}+c_{2} c_{6}\right) u v+p_{6}\left(f_{2} p_{6}-c_{2} c_{6}\right) v^{2}\right] u^{3} v^{3}, } \\
g= & {\left[\left(2 \alpha c_{6}+\alpha^{2} p_{6}-12 f_{2} c_{2}^{2}\right) u^{3}\right.} \\
& +\left(3 \alpha^{2} p_{6}^{2}+24 c_{2}^{2}\left(c_{2}^{4}-f_{2} p_{6}\right)+c_{6}\left(c_{6}+12 c_{2}^{3}+6 \alpha p_{6}\right)\right) u^{2} v \\
& +\left(3 \alpha^{2} p_{6}^{2}+2 c_{6}\left(6 c_{2}^{3}+c_{6}\right)+6 p_{6}\left(\alpha c_{6}-2 f_{2} c_{2}^{2}\right)\right) p_{6} u v^{2} \\
& \left.+p_{6}^{2}\left(c_{6}+p_{6} \alpha\right)^{2} v^{3}\right] \frac{u^{4} v^{5}}{12} \\
\Delta= & \frac{1}{16} u^{8} v^{9}\left(u+p_{6} v\right)^{3} \Delta_{\text {res }},
\end{aligned}
$$

with

$$
\begin{aligned}
\Delta_{\mathrm{res}} & =4 f_{2}^{3} u^{4}+(\ldots) u^{3} v+(\ldots) u^{2} v^{2}+(\ldots) u v^{3}+\frac{3 p_{6}\left(\alpha p_{6}+c_{6}\right)^{4}}{16} v^{4} \\
& =4 f_{2}^{3} \sigma^{4}+(\ldots) \sigma^{3} v+(\ldots) \sigma^{2} v^{2}+(\ldots) \sigma v^{3}+p_{6} c_{2}^{3} Q_{18} v^{4}
\end{aligned}
$$

where $\sigma=u+p_{6} v$ and $Q_{18}$ a degree 18 polynomial.

The six non-minimal points at $u=p_{6}=0$ with vanishing orders $(f, g, \Delta) \sim(4,6,12)$ are a result of the transverse intersections between the $\mathfrak{e}_{6}$, the $\mathfrak{s u}_{3}$ and the residual discriminant components. They are resolved by a blow-up at each point with one exceptional curve, over which the elliptic fiber turns out to be smooth. Thus these blow-ups do not introduce additional gauge algebras. They do however separate $\{u\}$ and $\{\sigma\}$ completely

\footnotetext{
${ }^{3}$ Note that the $\mathfrak{u}(1)$ charge here is normalized such that the only charged singlet has charge 1 . Compared to [24], this amounts to dividing the charge there by 2 .
} 
and lower the self-intersection number of the $\mathfrak{e}_{6}$ and $\mathfrak{s u}_{3}$ divisor both to 0 . The gauge anomaly is then cancelled by the six 27's at $u=\alpha p_{6}+c_{6}=0$ and $18 \times \mathbf{3}$ 's at $\sigma=Q_{18}=0$. Furthermore, the $\mathfrak{e}_{7}$ gauge anomaly is cancelled by the two $\frac{1}{2} \mathbf{5 6}$ 's at $v=f_{2}=0$. Finally, the gravitational anomaly requires 17 uncharged singlets, in agreement with the 21 free parameters in $f_{2}, c_{2}, c_{6}, p_{6}, \alpha$ modulo the four reparametrization degrees of freedom of the base $\mathbb{F}_{6}$. In order to obtain the F-theory geometry dual to the heterotic orbifold, we have to specialize these parameters further.

The first step towards the perturbative heterotic spectrum (4.1) should now be to ensure the correct tangential intersection between the $\mathfrak{e}_{6}$ divisor and the other discriminant components.

\subsection{Tangency of intersection and the need for a Type IV fiber}

Similar to the case of $\mathfrak{e}_{7}$, fundamental matter of $\mathfrak{e}_{6}$ generically appears at a tangential intersection of the $\mathfrak{e}_{6}$ locus with another discriminant component. While for $\mathfrak{e}_{7}$, the necessary order of tangency was three, it is four for $\mathfrak{e}_{6}$ (as can be seen from a generic Weierstrass model with $\left.E_{6}\right)$. However, for the $\mathrm{I}_{3}$ locus on $\left\{u+p_{6} v\right\}$, there is no possible factorization of $p_{6}$ such that one has an order four tangency at all points of intersection with $\{u\}$.

To resolve this issue, we propose that the $\mathfrak{s u}_{3}$ should be realized with a type IV fiber. First, the above naive picture applies to a single 7-brane hitting the $\mathfrak{e}_{6}$, such that the fundamental states can be thought of as massless strings ending on the $\mathfrak{e}_{6}$ branes and the single 7-brane. Locally, in the $\mathrm{I}_{3}$ version of $\mathfrak{s u}_{3}$, the $\mathfrak{e}_{6}$ sees three mutually local 7-branes which happen to pass through the same point. From the $\mathfrak{e}_{6}$ perspective, each of these branes would have to therefore meet at an order four tangency, which is impossible in the given setup. However, the type IV version of $\mathfrak{s u}_{3}$ is realized on four branes which are mutually non-local. Thus, there is the possibility of multi-pronged strings that end on two of the four branes while still constituting a single state from the $\mathfrak{e}_{6}$ perspective. Indeed, if one looks at a generic model with type IV $\mathfrak{s u}_{3}$ on $\{w\}$,

$$
f=w^{2} \tilde{f}, \quad g=w^{2} \tilde{g}^{2}, \quad \Delta=w^{4}(\underbrace{4 \tilde{f}^{3} w^{2}+27 \tilde{g}^{4}}_{\tilde{\Delta}}),
$$

one can see that the codimension two enhancement loci $\{w\} \cap\{\tilde{\Delta}\}=\{w=\tilde{g}=0\}$, which is known to host three hypermultiplets of triplets at each point, locally looks like two of the four branes on $\{w\}$ meeting the residual $\mathrm{I}_{1}$ locus $\{\tilde{\Delta}\}$ at quadratic order.

On the $\mathbb{F}_{6}$ base, such a non-transversal meeting is indeed possible between $\mathfrak{e}_{6}$ and type IV $\mathfrak{s u}_{3}$, both on +6 -curves: one simply has to set the $\mathfrak{s u}_{3}$ locus to be $\left\{u+p_{3}^{2} v\right\}$, which meets the $\mathfrak{e}_{6}$ locus on $\{u\}$ tangentially with order two. In the above model (4.2), setting $c_{2}=0$ enhances the singularity type over the $\mathfrak{s u}_{3}$ locus to type IV, which then is guaranteed to intersect the $\mathfrak{e}_{6}$ at quadratic order with $p_{6}=p_{3}^{2}$. The Weierstrass model now takes the form

$$
\begin{aligned}
f & =f_{2} u^{3} v^{3}\left(u+p_{3}^{2} v\right)^{2} \\
g & =\frac{1}{12} u^{4} v^{5}\left(u+p_{3}^{2} v\right)^{2}\left(\alpha\left(\alpha p_{3}^{2}+2 c_{6}\right) u+\left(c_{6}+\alpha p_{3}^{2}\right)^{2} v\right), \\
\Delta & =\frac{1}{16} u^{8} v^{9}\left(u+p_{3}^{2} v\right)^{4}\left(64 f_{2}^{3} u\left(u+p_{3}^{2} v\right)^{2}+3 v\left(\alpha\left(\alpha p_{3}^{2}+2 c_{6}\right) u+\left(c_{6}+\alpha p_{3}^{2}\right)^{2} v\right)^{2}\right) .
\end{aligned}
$$


Similar to the $\mathfrak{e}_{7} \times \mathfrak{s u}_{2}$ case, the tangency argument alone is not sufficient to obtain the correct orbifold dual model. Again, we can convince ourselves that despite the tuning $p_{6}=$ $p_{3}^{2}$, the non-minimal points at $p_{3}=u=0$, now with vanishing orders $(f, g, \Delta) \sim(5,6,12)$, are still resolved with six blow-ups (two at each point), with no additional gauge algebras or matter accompanying them (the intersection structure of these blow-ups do change). In fact, counting the singlets naively one finds that there are three singlets missing to cancel the gravitational anomaly. However, this is no surprise as we have set $c_{2}=0$ to obtain the type IV fiber, which does not change the charged spectrum on the tensor branch compared to the model with $\mathrm{I}_{3}$ (4.2). Therefore, the three parameters of $c_{2}$ still correspond to massless singlets present in the tensor branch theory.

\subsection{Enhancing the strongly coupled sector}

The next step of the tuning is now to enhance the gauge sector on the tensor branch, which becomes strongly coupled when we blow-down the exceptional curves. To do that, we again collapse all codimension two enhancement loci on the $\mathfrak{e}_{6}$ and $\mathfrak{s u}_{3}$ divisors on top of each other. A quick look at (4.5) reveals that the $\mathfrak{e}_{6}$ intersects the residual discriminant at $c_{6}+\alpha p_{3}^{2}=u=0$, while the $\mathfrak{s u}_{3}$ divisor meets it at $u+p_{3}^{2} v=0=c_{6}$. To collapse both loci onto $u=p_{3}=0$, we therefore have to tune $c_{6}=\beta p_{3}^{2}$. With some slight cosmetic redefinitions, ${ }^{4}$ our F-theory geometry is now given by

$$
\begin{aligned}
f & =f_{2} u^{3} v^{3}\left(u+p_{3}^{2} v\right)^{2} \\
g & =\frac{p_{3}^{2}}{12} u^{4} v^{5}\left(u+p_{3}^{2} v\right)^{2}\left(\mu u+\lambda^{2} p_{3}^{2} v\right), \\
\Delta & =\frac{1}{16} u^{8} v^{9}\left(u+p_{3}^{2} v\right)^{4}\left(64 f_{2}^{3} u\left(u+p_{3}^{2} v\right)^{2}+3 p_{3}^{4} v\left(\mu u+\lambda^{2} p_{3}^{2} v\right)^{2}\right) .
\end{aligned}
$$

Let us verify that this tuning indeed enhances the strongly coupled sector by blowing up the only non-minimal points at $u=p_{3}=0$, which now have vanishing orders $(f, g, \Delta) \sim$ $(5,9,15)$. The result of this process is a tree of five rigid curves for each point, cf. figure 3 . Inside there is a chain of $(-2)(-3)(-2)$ curves carrying singular fibers corresponding to the gauge algebras $\mathfrak{s u}_{2}-\mathfrak{s o}_{7}-\mathfrak{s u}_{2}$. Anomaly cancellation forces the matter content to be $\frac{1}{2}(\mathbf{2}, \mathbf{8}, \mathbf{1}) \oplus \frac{1}{2}(\mathbf{1}, \mathbf{8}, \mathbf{2})$. The blow-ups lower the self-intersection numbers of the $\mathfrak{e}_{6}$ divisor to -6 and of the $\mathfrak{s u}_{3}$ divisor to -3 , so they are non-higgsable, i.e., have no matter [53]. These blow-ups did not touch the $\mathfrak{e}_{7}$ loci, whose $2 \times \frac{1}{2} \mathbf{5 6}$ are localized at $\left\{v=f_{2}=0\right\}$. Finally, there are nine free parameters in $f_{2}, p_{3}, \lambda, \mu$, which after subtracting the four reparametrization degrees of freedom yield five uncharged singlets. The complete spectrum is therefore:

$$
\begin{aligned}
H_{\mathbf{1}_{0}} & =5 \\
H_{\text {charged }} & =\#\left(3 \times \frac{1}{2}((\mathbf{2}, \mathbf{8}, \mathbf{1}) \oplus(\mathbf{2}, \mathbf{8}, \mathbf{1}))+2 \times \frac{1}{2} \mathbf{5 6}\right)=104, \\
V & =133+78+8+3(3+21+3)=300, \\
T & =1+3 \cdot 5=16 \\
\Longrightarrow 273 & =H_{\text {charged }}+H_{\mathbf{1}_{0}}-V+29 T .
\end{aligned}
$$

\footnotetext{
${ }^{4}$ Define $\alpha \equiv \lambda-\sqrt{\lambda^{2}-\mu}$ and $\beta \equiv \sqrt{\lambda^{2}}-\mu$.
} 

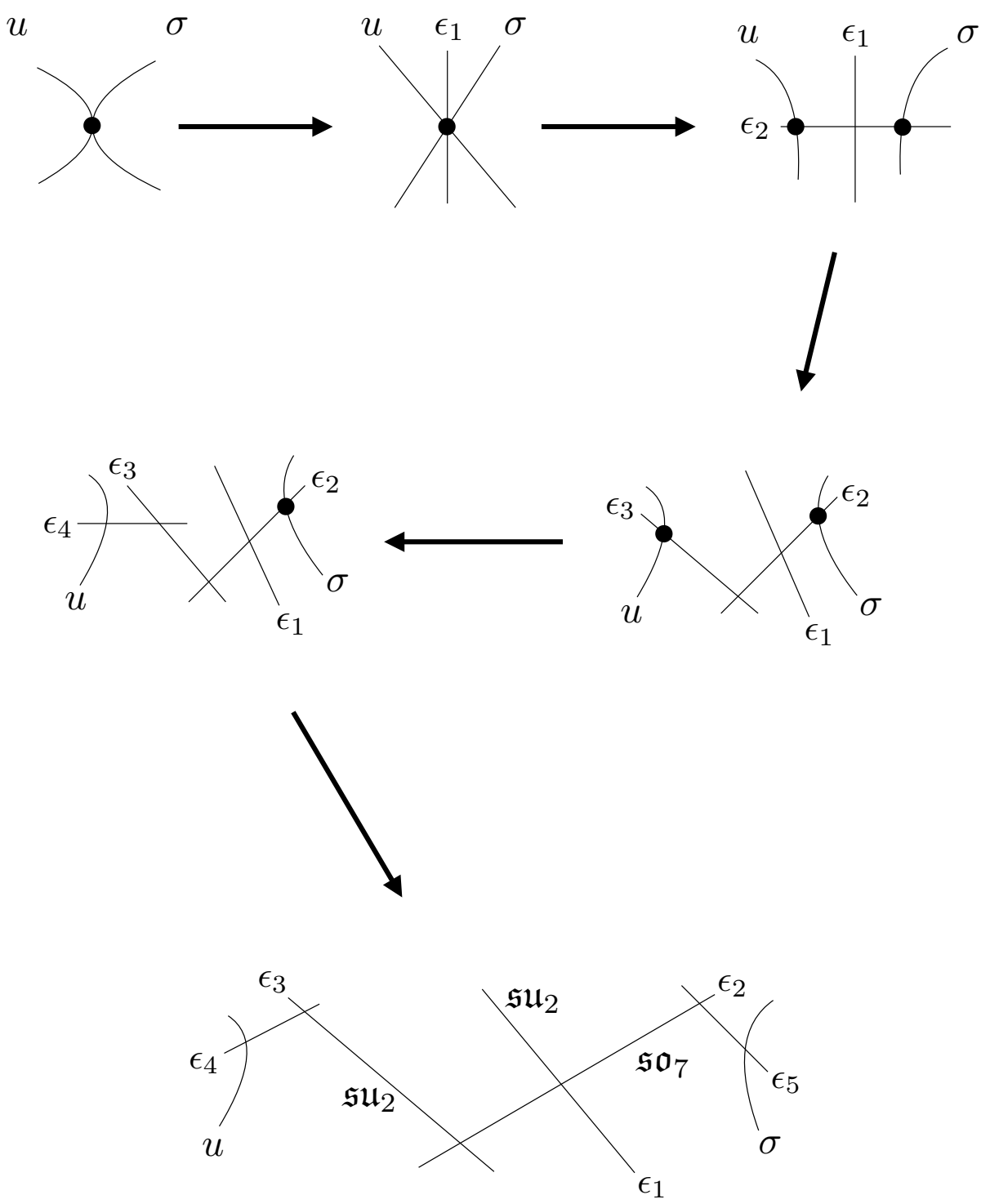

Figure 3. Blowing-up the base at the three points $u=p_{3}=0$ where the $\mathfrak{e}_{6}$ divisor $\{u\}$ and the $\mathfrak{s u}_{3}$ divisor $\left\{\sigma \equiv u+p_{3}^{2} v\right\}$ intersect. It requires five $\mathbb{P}^{1}$ s $\left\{\epsilon_{i}\right\}$ to resolve all non-minimal points (black dots). After the blow-ups the curve $\{u\}$ has self-intersection -6 , and $\{\sigma\}$ has -3 . The sequence of curves $\epsilon_{1}-\epsilon_{2}-\epsilon_{3}$ has self-intersections $(-2,-3,-2)$ and have the gauge algebras $\mathfrak{s u}_{2}-\mathfrak{s o}_{7}-\mathfrak{s u}_{2}$. At the two intersection points we have $\frac{1}{2}(\mathbf{8}, \mathbf{2})$, where $\mathbf{8}$ is the spinor representation of $\mathfrak{s o}_{7}$.

It is reassuring to see that the singlets are counted correctly by our free parameters, as indicated by the cancellation of the gravitational anomaly.

We can analyze further aspects of the strongly coupled sector by decompactifying the curves supporting the $\mathfrak{e}_{6}$ and $\mathfrak{s u}_{3}$ gauge groups. Doing so, we are left with the tensor branch of a 6D SCFT (actually three copies) with tensor branch description (with notation as in references $[31,32,47])$ :

$$
\left[E_{6}\right], 1, \stackrel{\mathfrak{s u}_{2}}{2}, \underset{1}{\stackrel{\mathfrak{s o}_{7}}{3}}, \stackrel{\mathfrak{s u}_{2}}{2}
$$


Namely at the SCFT point, there is an emergent $\mathrm{SO}(9)$ flavor symmetry. Indeed, the -1 curve theory supports an E-string theory, and we can gauge the $\mathfrak{s o}_{7}$ factor of the $\mathfrak{s o}_{7} \times \mathfrak{s o}_{9} \subset \mathfrak{e}_{8}$ subalgebra. The $\mathfrak{s u}_{3}$ gauge symmetry of the compact model is obtained by gauging a subalgebra of this $\mathrm{SO}(9)$ flavor symmetry.

Cross check - Dual heterotic $\mathbf{K} 3$ as $\mathbb{Z}_{\mathbf{3}}$-orbifold. At this point, it is worth comparing the above tuning, motivated solely from an F-theory perspective, with the heterotic K3 in the dual orbifold model. For the case at hand, the elliptic fibration of the K3 has to have three $\mathrm{IV}^{*}$ fibers to be compatible with the $T^{4} / \mathbb{Z}_{3}$ orbifold (see e.g. [58]). This means that the Weierstrass functions have to be of the form $\left(f_{\mathrm{K} 3}, g_{\mathrm{K} 3}\right)=\left(0, q_{3}^{4}\right)$, where the position of the singular fibers are the roots of some degree three polynomial $q_{3}$. Furthermore, the heterotic dual of an $\mathrm{F}$-theory model over $\mathbb{F}_{6}$ has 18 mobile instantons, which again have to be localized at the orbifold fixed points, i.e., inside the $\mathrm{IV}^{*}$ fibers.

Naively, these conditions seems to be incompatible with (4.5): identifying $f_{\mathrm{K} 3}$ as the coefficient of $u^{4} v^{4}$ in $f$ would then require setting $2 f_{2} p_{3}^{2}$ to 0 . However, by setting either of the two functions to zero would enhance the singularity type over either $\{v\}$ or $\{u\}$, none of which would yield the spectrum of the heterotic orbifold.

On the other hand, the curve $\{u\}$ is not special on the $\mathbb{F}_{6}$ base of F-theory. Unlike the -6-curve $\{v\},+6$-curves come in a family $\left\{s \equiv u+q_{6} v=0\right\}$ for any $q_{6}$. Therefore, there is no obvious reason why one cannot identify the gluing K3 along any of these +6 -curves $\{s\}$. In fact, one might view this shift by $q_{6}$ as a consequence of the non-trivial shift inside both $E_{8}$ 's on the heterotic orbifold side. So we write the F-theory Weierstrass functions (4.5) as polynomials $f=\sum_{k} f_{d_{k}}^{\prime} s^{k} v^{5-k}, g=\sum_{k} g_{d_{k}}^{\prime} s^{k} v^{8-k}$. Then, the heterotic K3 is the Weierstrass model with

$$
f_{\mathrm{K} 3} \equiv f_{8}^{\prime}=f_{2}\left(2 p_{3}^{2}-5 q_{6}\right)
$$

The necessary condition $f_{\mathrm{K} 3}=0$ can now be solve with $q_{6}=2 / 5 p_{3}^{2}$. It is reassuring to see that the resulting $g_{\mathrm{K} 3}$ as well as the polynomial $g_{18}^{\prime}$ encoding the location of the 18 mobile instantons are compatible with the orbifold:

$$
\begin{aligned}
g_{\mathrm{K} 3} \equiv g_{12}^{\prime} & =\frac{\left(5 \lambda^{2}-4 \mu\right)}{60} p_{3}^{4}, \\
g_{18}^{\prime} & =-\frac{10 \lambda^{2}+11 \mu}{300} p_{3}^{6},
\end{aligned}
$$

i.e., the heterotic $\mathrm{K} 3$ has three $\mathrm{IV}^{*}$ fibers at $p_{3}=0$, at which the 18 instantons are localized.

\subsection{Engineering the $\mathfrak{u}(1)$ and singlet counting}

The only part of the gauge group that is still missing is the $\mathfrak{u}(1)$ factor. To engineer it, the F-theory fibration has to have a non-trivial Mordell-Weil group, i.e., another rational section in addition to the zero section. With the tuned model (4.6), this turns out to be fairly easy. By inspection of $g$, we see that it is almost a perfect square up to the factor 
$v^{5}\left(\mu u+\lambda^{2} p_{3}^{2} v\right)$. However, this can be easily changed by setting $\mu=0$, yielding

$$
\begin{aligned}
f & =f_{2} u^{3} v^{3}\left(u+p_{3}^{2} v\right)^{2}, \\
g & =\frac{\lambda^{2}}{12} p_{3}^{4} u^{4} v^{6}\left(u+p_{3}^{2} v\right)^{2}, \\
\Delta & =\frac{1}{16} u^{8} v^{9}\left(u+p_{3}^{2} v\right)^{4}\left(64 f_{2}^{3} u\left(u+p_{3}^{2} v\right)^{2}+3 \lambda^{4} p_{3}^{8} v^{2}\right) .
\end{aligned}
$$

Then, the Weierstrass equation

$$
y^{2}=x^{3}+f x+g
$$

has a rational solution at $(x, y)=(0, \sqrt{g})=\left(0, \lambda p_{3}^{2} u^{2} v^{3}\left(u+p_{3}^{2} v\right) / \sqrt{12}\right)$, which is clearly not the zero-section at infinity.

Setting $\mu=0$ makes the singularity over the locus $v=f_{2}=0$ non-minimal. The blow-up introduces two tensors and no additional gauge group. They do, however, change the self-intersection of the $\mathfrak{e}_{7}$ divisor to -8 , which does not allow for any $\mathfrak{e}_{7}$ matter. The change in the gravitational anomaly can also be computed:

$$
\delta H-\delta V+29 \delta T=(-56-1)-1+2 \cdot 29=0 .
$$

Unfortunately, this tuning is not enough to produce the heterotic dual, as reflected by the mismatch in the number of uncharged singlets: by losing only one singlet compared to (4.6), we still have four uncharged singlets, which exceeds the counting in the heterotic spectrum (4.1) by two.

To resolve this paradox, we need to pay more attention to the Higgs branch of the heterotic model. Given the heterotic spectrum (4.1) with gauge algebra $\mathfrak{e}_{6} \times \mathfrak{s u}_{3} \times \mathfrak{e}_{7} \times \mathfrak{u}(1)$, one cannot higgs the $\mathfrak{u}(1)$ in a D-flat manner with just one charged singlet. Consequently, any higgsing that breaks the $\mathfrak{u}(1)$ must proceed via vevs of the other matter multiplets, which necessarily also break parts of the non-abelian gauge group. Conversely, this means that an honest complex structure tuning that corresponds to a chain of unhiggsing should have implemented the $\mathfrak{u}(1)$ before the appearance of all the non-abelian gauge factors.

One starting point could be a model with $\mathfrak{e}_{6} \times \mathfrak{e}_{7}$ gauge symmetry only, given by the Weierstrass model

$$
\begin{aligned}
f & =u^{3} v^{3} \tilde{f}, \quad g=u^{4} v^{5} \tilde{g}, \quad \text { with } \\
\tilde{f} & =f_{2} u^{2}+f_{8} u v+f_{14} v^{2}, \\
\tilde{g} & =g_{6} u^{3}+g_{12} u^{2} v+g_{18} u v^{2}+\gamma_{12}^{2} v^{3} \\
\Longrightarrow \Delta & =u^{8} v^{9}\left(4 u^{7} f_{2}^{3}+27 v^{7} \gamma_{12}^{4}+u v(\ldots)\right),
\end{aligned}
$$

which certainly lies in the Higgs branch of the heterotic model (one can break the $\mathfrak{s u}_{3} \times \mathfrak{u}(1)$ with the charged triplets only). This geometry was also be taken as the starting point of our tuning process, that led us to the above $\mathfrak{e}_{6} \times \mathfrak{s u}_{3} \times \mathfrak{e}_{7}$ model (4.6) (see appendix A). Thus one might hope that by first implementing the additional rational section, one could still incorporate the above tuning to yield the desired $\mathfrak{s u}_{3}$ part. Unfortunately, despite the many 
recent advances in the study of $\mathfrak{u}(1)$ 's in F-theory, ${ }^{5}$ it is technically still very challenging to come up with a model that not only includes the $\mathfrak{u}(1)$, but also has no redundancies such that we can correctly count the uncharged singlets by the free parameters in the Weierstrass model. ${ }^{6}$

However, there is one key feature of the $\mathfrak{u}(1)$ which is easy to implement and sufficient to identify one necessary complex structure tuning. Starting from (4.14) and enhancing the gauge group to $\mathfrak{e}_{6} \times \mathfrak{e}_{7} \times \mathfrak{u}(1)$, the two $\frac{1}{2} \mathbf{5 6}$ hypermultiplets have to merge into a single hypermultiplet charged under the $\mathfrak{u}(1)$. Since such a spectrum is perfectly compatible with minimal Kodaira fiber types, standard F-theory dictates that a necessary geometric condition for the existence of such a $\mathfrak{u}(1)$ is the factorization $f_{2} \rightarrow f_{1}^{2}$, which combines the two loci of the $\frac{1}{2} \mathbf{5 6}$ 's in the $\mathfrak{e}_{6} \times \mathfrak{e}_{7}$ model (4.14). Since none of the subsequent tunings to obtain the type IV $\mathfrak{s u}_{3}$ affect $f_{2}$, the factorization should also persist in the result (4.11).

The factorization of $f_{2} \rightarrow f_{1}^{2}$ reduces the number of free parameters in (4.11) by one. Another one can be removed by noticing that the parameter $\lambda$ can be absorbed by a rescaling of $(u, v) \rightarrow \lambda^{-1 / 6}(u, v)$ and a redefinition of $f_{1} \rightarrow f_{1} \lambda^{2 / 3}$. With that, the resulting fibration

$$
\begin{aligned}
f & =f_{1}^{2} u^{3} v^{3}\left(u+p_{3}^{2} v\right)^{2}, \\
g & =\frac{1}{12} p_{3}^{4} u^{4} v^{6}\left(u+p_{3}^{2} v\right)^{2}, \\
\Delta & =\frac{1}{16} u^{8} v^{9}\left(u+p_{3}^{2} v\right)^{4}\left(64 f_{1}^{6} u\left(u+p_{3}^{2} v\right)^{2}+3 p_{3}^{8} v^{2}\right),
\end{aligned}
$$

now has the right number of singlets. Therefore, F-theory compactified on (4.15) should be dual to the heterotic $\mathbb{Z}_{3}$-orbifold with the spectrum (4.1). Just like in the $\mathfrak{e}_{7} \times \mathfrak{s u}_{2}$ case, the single $(\mathbf{2 7}, \mathbf{3})$ hypermultiplet is spread across three points on the base. Hence, their appearance cannot be just a local phenomenon and should be further investigated in future work.

As a final consistency check, let us comment on the global structure of the gauge group of this model. From the spectrum (4.1), we see that the $\mathfrak{u}(1)$ charges of the matter states are tied to their non-abelian representations in a particular way:

- The $\mathfrak{u}(1)$ is normalized such that the singlet has charge 1 .

- Fundamentals under $\mathfrak{e}_{7}$ have half-integer charges.

- Fundamentals under $\mathfrak{e}_{6}$ and $\mathfrak{s u}_{3}$ have charges in multiples of $\frac{1}{3}$; in the case of triplets, the charges are $\frac{2}{3} \bmod \mathbb{Z}$.

- The bifundamental $(\mathbf{2 7}, \mathbf{3})$ has integer charge.

\footnotetext{
${ }^{5}$ Starting with [59] and subsequently [60-62] there have been many recent developments. For a more complete set of references, see the review article [63].

${ }^{6}$ For example, while the so-called Morrison-Park model [60] guarantees a section by embedding the fiber into an $\mathrm{Bl}_{1} \mathbb{P}_{112}$ ambient space, the resulting Weierstrass model has some redundancies in terms of the coefficients of the $\mathrm{Bl}_{1} \mathbb{P}_{112}$-hypersurface, which have to be identified for a correct counting of uncharged singlets.
} 
The associated gauge group thus has to be

$$
\frac{E_{6} \times \mathrm{SU}(3) \times E_{7} \times \mathrm{U}(1)}{\mathbb{Z}_{6}}=\frac{E_{6} \times \mathrm{SU}(3) \times E_{7} \times \mathrm{U}(1)}{\mathbb{Z}_{3} \times \mathbb{Z}_{2}} .
$$

In F-theory, such non-trivial global gauge group structures are realized whenever the zero section and independent section intersect the codimension one fibers over the gauge divisor at different components in the resolved geometry [11]. ${ }^{7}$ For the three gauge algebras at hand, there is (up to automorphisms of the respective Dynkin diagrams) only one possibility, which all shrink in the singular limit. Thus, the independent section has to pass through the singular point over the gauge divisors. Since in the model (4.15), both $f$ and $g$ vanish over these codimension one loci $\left(\{u\},\{v\},\left\{u+p_{3} v^{3}\right\}\right)$, the singular point of the elliptic fiber sits and $x=y=0$, which is indeed intersected by the section $(x, y)=(0, \sqrt{g})$. Therefore, we see that our tuned F-theory geometry also accommodates the global gauge group structure of the heterotic orbifold.

\subsection{Adding more bifundamentals}

Much as in the case of the $\mathfrak{e}_{7} \times \mathfrak{s u}_{2}$ model, we ask whether it is possible to engineer $\mathfrak{e}_{6} \times \mathfrak{s u}_{3}$ gauge theories with more than one "exotic" bifundamental. Locally, at least, there seems to be little issue with this procedure, provided we relax the constraints on the self-intersection numbers of our curves. For example, we can simply add more zeroes to produce additional delocalized bifundamentals.

Focussing on the purely gauge theoretic aspects of $6 \mathrm{D}$ anomaly cancellation, the relevant constraints are (see e.g. $[51,55])$ :

$$
\begin{aligned}
\left(D_{\mathfrak{e}_{6}} \cdot D_{\mathfrak{e}_{6}}+6\right) & =N_{(\mathbf{2 7}, \mathbf{1})}+3 \times N_{(\mathbf{2 7}, \mathbf{3})} \\
\left(6 D_{\mathfrak{s u}(3)} \cdot D_{\mathfrak{s u}(3)}+18\right) & =N_{(\mathbf{1 , 3})}+27 \times N_{(\mathbf{2 7 , 3})} \\
D_{\mathfrak{e}_{6}} \cdot D_{\mathfrak{s u}(3)} & =6 \times N_{(\mathbf{2 7}, \mathbf{3})} .
\end{aligned}
$$

where here, $D_{\mathfrak{g}}$ denotes the divisor class of a curve wrapped by a seven-brane with gauge algebra $\mathfrak{g}$, and the $N$ 's indicate the multiplicity of various representations.

Increasing the number of bifundamentals necessarily raises the self-intersection number relative to the case of a single bifundamental. As an example with more than one bifundamental which satisfies these conditions, we can take:

$$
\begin{aligned}
& N_{(\mathbf{2 7}, \mathbf{3})}=2, \quad N_{(\mathbf{2 7}, \mathbf{1})}=0, \quad N_{(\mathbf{1}, \mathbf{3})}=0, \\
& D_{\mathfrak{e}_{6}} \cdot D_{\mathfrak{e}_{6}}=0, \quad D_{\mathfrak{s u}_{3}} \cdot D_{\mathfrak{s u}(3)}=6, \quad D_{\mathfrak{e}_{6}} \cdot D_{\mathfrak{s u}(3)}=12 .
\end{aligned}
$$

It seems plausible that this could arise in a compact geometry, but again, we defer a full analysis to future work.

\footnotetext{
${ }^{7}$ In this reference, the derivation of the global gauge group structure assumes a smooth global geometry, which we have not provided here. Given the presence of the non-minimal point, a Calabi-Yau resolution of the tuned Weierstrass model without blow-ups in the base will most likely include non-flat fibers. However, we expect the statement of [11] to still hold, since the global group structure only depends on the interplay between the rational sections and codimension one fibers, which in our model are still of Kodaira-Tate form.
} 


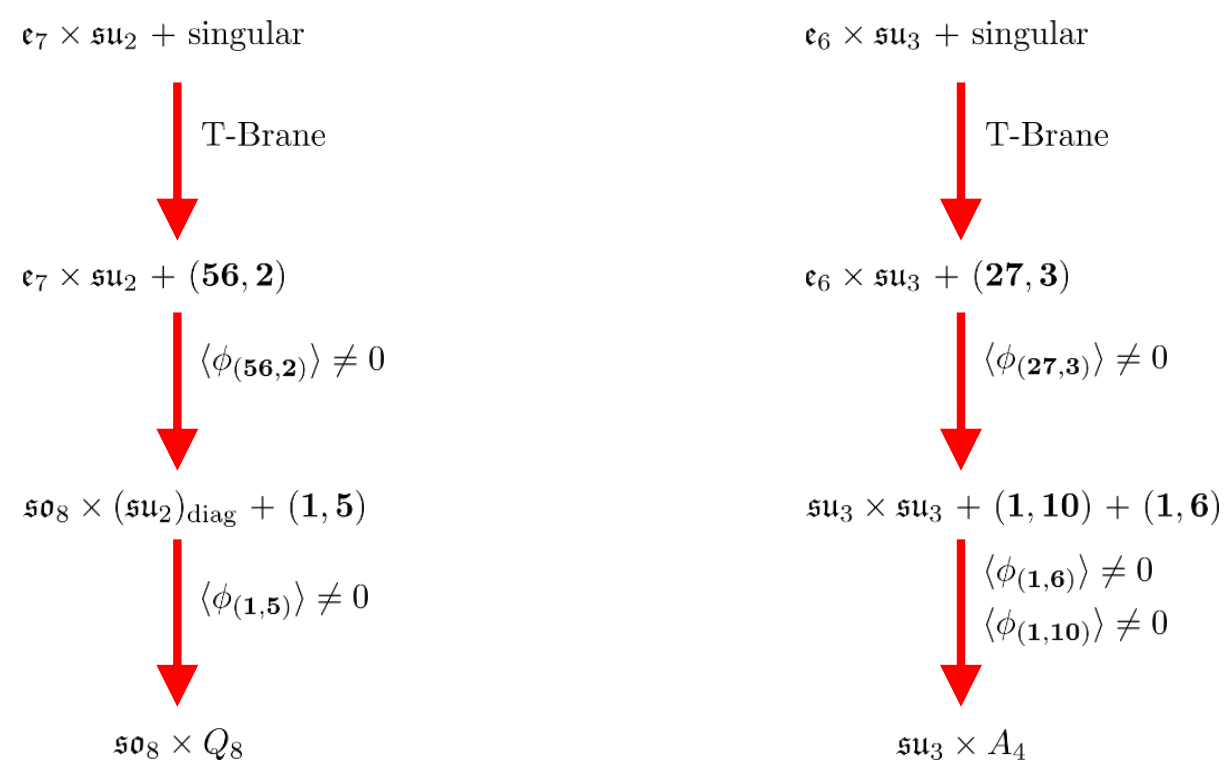

Figure 4. Depiction of breaking patterns associated with vevs for exotic bifundamentals. Here, we assume that the continuous symmetries are flavor symmetries so that no D-term constraints need to be satisfied. The final stage of the breaking patterns leads to a finite order non-abelian group. Here, $Q_{8}$ denotes the order eight quaternion group and $A_{4}$ the alternating group on four letters. In the case of the breaking pattern for $\mathfrak{e}_{6} \times \mathfrak{s u}_{3}$ to $\mathfrak{s u}_{3} \times A_{4}$, this also requires a vev for a scalar in the $(\mathbf{1}, \mathbf{6})$ of $\mathfrak{s u}_{3} \times \mathfrak{s u}_{3}$, which descends from the $(\mathbf{2 7}, \mathbf{1})$ of $\mathfrak{e}_{6} \times \mathfrak{s u}_{3}$.

Much as in the case of $\mathfrak{e}_{7} \times \mathfrak{s u}_{2}$ exotic bifundamentals, we can compactify our 6D hypermultiplet on a genus $g$ curve, and in so doing, produce $g 4 \mathrm{D} \mathcal{N}=1$ chiral multiplets in the $(\mathbf{2 7}, \mathbf{3})$ representation of $\mathfrak{e}_{6} \times \mathfrak{s u}_{3}$, and $g$ in the conjugate representation. Again, this discussion neglects subtleties having to do with embedding the matter curves in a Calabi-Yau fourfold geometry, or the presence of ambient fluxes.

\section{Breaking patterns}

In the previous sections, we focused on the geometric realization of exotic bifundamental matter field. From this starting point, a number of additional exotic structures can be realized in F-theory. The main idea is that by activating background vevs for bifundamental matter fields, we can reach unbroken symmetries with both high dimension representations of lower rank symmetry groups, as well as finite non-abelian discrete symmetries. This follows the general strategy advocated in reference [21] to get non-abelian finite groups from breaking patterns of a continuous symmetry group. See figure 4 for a depiction of the breaking patterns associated with the exotic bifundamentals $(\mathbf{5 6}, \mathbf{2})$ of $\mathfrak{e}_{7} \times \mathfrak{s u _ { 2 }}$ and the $(27,3)$ of $\mathfrak{e}_{6} \times \mathfrak{s u}_{3}$.

Now, in the specific geometries we have constructed, we have a single bifundamental hypermutiplet between two gauge groups factors. Activating a vev in this case is obstructed because of the D-term constraints from the two gauge algebras. The reason comes down to the fact that to actually Higgs a $\mathfrak{s u}_{N} \times \mathfrak{g}$ gauge theory with matter fields in the fundamental 
representation, we need at least two hypermultiplets (see e.g [53]). ${ }^{8}$ Of course, we can take a decompactification limit where all gauge group factors turn into flavor symmetry factors. In this limit (which is also decoupled from gravity), there are no D-term constraints to satisfy, and we can reduce our problem to a purely group theoretic exercise. If we insist on a model where all symmetries are gauged, we can also contemplate models with more than one exotic bifundamental. As we have indicated in previous sections, there appears to be no a priori obstruction to realizing $6 \mathrm{D}$ vacua with this feature, and even fewer constraints for $4 \mathrm{D}$ vacua.

With this in mind, our aim here will be to explore the breaking patterns generated by giving a vev to an exotic bifundamental. For ease of exposition, we focus on the special case where the pair of gauge algebras are decoupled, namely we treat them as global symmetries. Note that since the exotic bifundamentals are delocalized across multiple points of the geometry, we must also keep all such singular points localized in a single patch of the geometry.

Of course, we can also weakly gauge these flavor symmetries (and introduce a corresponding tensor multiplet to cancel gauge anomalies) and obtain similar breaking patterns in models where there is more than one exotic bifundamental. As we have already remarked, the explicit geometric realization of these cases is likely to be more involved, though a priori, there does not appear to be any bottom up obstruction to realizing such a model.

Our plan in the rest of this section will be to study the resulting breaking patterns from the $(\mathbf{5 6}, \mathbf{2})$ of $\mathfrak{e}_{7} \times \mathfrak{s u}_{2}$ and the $(\mathbf{2 7}, \mathbf{3})$ of $\mathfrak{e}_{6} \times \mathfrak{s u}_{3}$.

\subsection{The $(56,2)$ of $\mathfrak{e}_{7} \times \mathfrak{s u}_{2}$}

As a first example, consider the breaking pattern generated by a single $(\mathbf{5 6}, \mathbf{2})$ of $\mathfrak{e}_{7} \times \mathfrak{s u}_{2}$. Returning to our discussion in appendix B.1, we observe that there is a singlet in the decomposition to the subalgera $\mathfrak{s o}_{8} \times\left(\mathfrak{s u}_{2}\right)_{\text {diag: }}$ :

$$
\begin{aligned}
& \mathfrak{e}_{7} \times \mathfrak{s u}_{2} \supset \mathfrak{s o}_{8} \times\left(\mathfrak{s u}_{2}\right)_{\text {diag }} \\
&(\mathbf{5 6}, \mathbf{2}) \rightarrow\left(\boldsymbol{8}_{v}, \mathbf{3}\right)+\left(\mathbf{8}_{s}, \mathbf{3}\right)+\left(\mathbf{8}_{c}, \mathbf{3}\right) \\
&+\left(\mathbf{8}_{v}, \mathbf{1}\right)+\left(\mathbf{8}_{s}, \mathbf{1}\right)+\left(\mathbf{8}_{c}, \mathbf{1}\right) \\
&+(\mathbf{1}, \mathbf{5})+(\mathbf{1}, \mathbf{3})^{\oplus 3}+(\mathbf{1}, \mathbf{1})^{\oplus 2} .
\end{aligned}
$$

Note also that the coset space $\mathfrak{e}_{7} \times \mathfrak{s u}_{2} / \mathfrak{s o}_{8} \times\left(\mathfrak{s u}_{2}\right)_{\text {diag }}$ has dimension 105 , the same as the Goldstone modes:

$$
\begin{aligned}
\text { Goldstone Modes } & :\left(\mathbf{8}_{v}, \mathbf{3}\right)+\left(\mathbf{8}_{s}, \mathbf{3}\right)+\left(\mathbf{8}_{c}, \mathbf{3}\right) \\
+ & \left(\mathbf{8}_{v}, \mathbf{1}\right)+\left(\mathbf{8}_{s}, \mathbf{1}\right)+\left(\mathbf{8}_{c}, \mathbf{1}\right)+(\mathbf{1}, \mathbf{3})^{\oplus 3} .
\end{aligned}
$$

\footnotetext{
${ }^{8}$ To see why this is so, it is enough to consider the super-Higgs mechanism for $\mathfrak{s u}{ }_{N}$ gauge theory. Suppose we have a theory of $M$ hypermultiplets in the fundamental representation. Writing the collection of scalars as pairs of complex scalars $q_{I}$ and $\widetilde{q}_{I}$ for $I=1, \ldots, M$ such that each doublet $\left(q_{I}, \widetilde{q}_{I}^{\dagger}\right)$ rotates as a doublet under $\mathrm{SU}(2)$ R-symmetry, a breaking pattern of $\mathfrak{s u}_{N}$ requires us to give a vev to two hypermultipets. Moreover, as can be seen from the way the Goldstone bosons are eaten, we need one set of complex scalars transforming in the fundamental representation, and one set of complex scalars in the conjugate representation to get a vev. This is problematic when we have a single bifundamental, since then, only $q$ or $\widetilde{q}$ can be eaten.
} 
Nevertheless, as we have already remarked, the D-flatness conditions cannot be satisfied with a single exotic bifundamental. Taking this into acount, we see that the leftover modes not parameterizing the coset includes the $(\mathbf{1}, \mathbf{5})$ of $\mathfrak{s o}_{8} \times \mathfrak{s u}(2)_{\text {diag. }}$. This is a highdimensional representation, and as we have remarked, it has proven difficult to engineer string compactifications with this representation, though as far as we are aware there is no "no-go" theorem forbidding the appearance of these representations (see, however, [12]). Indeed, the present discussion suggests that it ought to be possible to find models with more than one exotic bifundamental, and thus higgs further to such an exotic representation.

This also provides a way for us to generate a high order non-abelian discrete group. To see why, we note that a vev for the $\mathbf{5}$ of $\left(\mathfrak{s u}_{2}\right)_{\text {diag }}$ leaves unbroken the order eight group known as $Q_{8}$, the quaternion group [48]. ${ }^{9}$

\subsection{The $(27,3)$ of $\mathfrak{e}_{6} \times \mathfrak{s u}_{3}$}

As another example, consider the breaking pattern generated by a single $(\mathbf{2 7}, \mathbf{3})$ of $\mathfrak{e}_{6} \times \mathfrak{s u}_{3}$. Returning to our discussion in appendix B.2, we observe that there is a singlet in the decomposition to the subalgebra $\left(\mathfrak{s u}_{3}\right)_{(3)} \times\left(\mathfrak{s u}_{3}\right)_{\text {diag: }}$ :

$$
\begin{aligned}
\mathfrak{e}_{6} \times \mathfrak{s u}_{3} & \supset\left(\mathfrak{s u}_{3}\right)_{(3)} \times\left(\mathfrak{s u}_{3}\right)_{\operatorname{diag}} \\
(\mathbf{2 7}, \mathbf{3}) & \rightarrow(\mathbf{1}, \mathbf{8})^{\oplus 2}+(\mathbf{1}, \mathbf{1})+(\mathbf{1}, \mathbf{1 0})+(\mathbf{3}, \mathbf{8})+(\mathbf{3}, \mathbf{1})+(\overline{\mathbf{3}}, \mathbf{8})+(\overline{\mathbf{3}}, \mathbf{1}) .
\end{aligned}
$$

Note also that the coset space $\mathfrak{e}_{6} \times \mathfrak{s u}_{3} /\left(\mathfrak{s u}_{3}\right)_{(3)} \times\left(\mathfrak{s u}_{3}\right)_{\text {diag }}$ has dimension 70 , the same as the Goldstone modes:

$$
\text { Goldstone Modes: }(\mathbf{1}, \mathbf{8})^{\oplus 2}+(\mathbf{3}, \mathbf{8})+(\mathbf{3}, \mathbf{1})+(\overline{\mathbf{3}}, \mathbf{8})+(\overline{\mathbf{3}}, \mathbf{1}) \text {, }
$$

which leaves us with a matter field in the $\mathbf{1 0}$ of $\mathfrak{s u}_{3}$. This is the three-index symmetric representation of $\mathfrak{s u}_{3}$, and has been viewed as difficult to engineer in F-theory [12]. Here, we see that if there really is an issue, it cannot be ascribed to local geometric conditions but must instead have to do with subtle global questions about compact Calabi-Yau threefolds.

Giving a further vev to this uneaten mode results in a continuous group [49], but in tandem with a vev for matter in the $\mathbf{6}$ of $\mathfrak{s u}_{3}$, all continuous symmetries are broken. Note that the decomposition of the fundamental of $\mathfrak{e}_{6}$ contains such a representation:

$$
\begin{aligned}
\mathfrak{e}_{6} \times \mathfrak{s u}_{3} & \supset\left(\mathfrak{s u}_{3}\right)_{(3)} \times\left(\mathfrak{s u}_{3}\right)_{\mathrm{diag}} \\
(\mathbf{2 7}, \mathbf{1}) & \rightarrow(\mathbf{1}, \mathbf{6})+(\mathbf{1}, \overline{\mathbf{3}})+(\mathbf{3}, \overline{\mathbf{3}})+(\overline{\mathbf{3}}, \overline{\mathbf{3}}) .
\end{aligned}
$$

The combination of a vev in the $\mathbf{1 0}$ and $\mathbf{6}$ of $\mathfrak{s u}_{3}$ breaks the continuous group to $A_{4}$ the alternating group on four letters [49]. Note that we typically have many matter fields in the $(\mathbf{2 7}, \mathbf{1})$. From this perspective, the primary challenge in realizing compact models will, as stated before, be to obtain models with more than one exotic bifundamental.

\footnotetext{
${ }^{9}$ Recall that $Q_{8}$ is the group with relations $\left\langle\bar{e}, i, j, k \mid \bar{e}^{2}=e, i^{2}=j^{2}=k^{2}=i j k=\bar{e}\right\rangle$, with $e$ the identity. This group has order eight.
} 


\section{Conclusions}

F-theory is a remarkably flexible framework for realizing a broad class of low energy effective field theories via string compactification. From this perspective, it is important to understand the list of possible exotic phenomena involving singular limits of moduli in such backgrounds. Such singular limits can lead to novel structures in low energy effective field theory, including high dimension representations for matter multiplets, as well as non-abelian discrete symmetries. In this paper we have taken some preliminary steps in developing a systematic framework for the construction of "exotic" bifundamental matter, as well as the subsequent symmetry breaking patterns generated by such matter fields. From a geometric perspective, the main idea in our approach is to allow the appearance of singular elliptically fibered Calabi-Yau threefolds in which the multiplicity of vanishing at a collision of two components of the discriminant locus is at least a $(4,6,12)$ point. When this collision has a sufficiently high order of tangency, there are additional directions in the Higgs branch of moduli space, and it is possible to activate a T-brane deformation, moving away from the SCFT point, and instead to a weakly coupled gauge theory. This new point in moduli space is described by the same singular Calabi-Yau geometry, but is non-singular in the physics description. We have illustrated these general considerations in the special case of bifundamental matter for $\mathfrak{e}_{7} \times \mathfrak{s u}_{2}$ and $\mathfrak{e}_{6} \times \mathfrak{s u}_{3}$, matching them to explicit dual heterotic orbifold models. Additionally, further higgsing of these theories leads to matter in "exotic" high dimension representations of $\mathfrak{s u}_{2}$ and $\mathfrak{s u}_{3}$. In the remainder of this section we discuss some avenues of further investigation.

One of the items left open by our analysis is a general criterion for the order of tangency needed in order to activate a T-brane deformation to a weakly coupled gauge theory. Rather, we have used a combination of top down and bottom up considerations to provide plausible candidates for such a match. In particular, we have often relied on some global features of heterotic/F-theory duality, even though the appearance of exotic bifundamental matter appears to be an issue which is localized in the geometry of an F-theory compactification. Developing a concise description of general necessary and sufficient conditions to realize such exotic bifundamental matter would seem a worthwhile undertaking. Specifically, the feature that the bifundamental hypermultiplet is spread across several points deserves further investigation. A better understanding of this phenomenon could provide us with methods to construct duals of other orbifolds with interesting matter (e.g., $\mathbb{Z}_{3}$ orbifolds with the three-index antisymmetric $(\mathbf{8 4})$ of $\left.\mathfrak{s u}_{9}\right)$, and to extend our analysis to F-theory vacua which do not have heterotic duals.

We have also presented evidence that we can engineer discrete non-abelian symmetries in F-theory by performing an appropriate higgsing with such exotic bifundamental matter. We have deferred an explicit construction of models with more than one hypermultiplet in a bifundamental representation, but as we have already remarked, there does not appear to be any issue with engineering such examples from the perspective of bottom up considerations. Further, a geometric characterization of non-abelian discrete symmetries in F-theory remains an important open problem to understand better.

Along these lines, it would be interesting to better understand in systematic terms the class of discrete symmetries which can actually be realized in string compactification. The 
present method using exotic bifundamental matter provides some examples, but it would be interesting to further analyze whether there are sharp upper bounds on the order, structure and representations of such delicate objects.

Another important direction to explore further is the behavior of such exotic bifundamental matter upon compactification to four dimensions. One of the standard tools for understanding chiral matter spectra in particular involves coupling hypermultiplets localized on matter curves to various gauge field and bulk fluxes. Due to the way these matter fields are engineered in F-theory, it would be interesting to examine the conditions necessary to realize a chiral matter spectrum for them. Turning the discussion around, this may also provide insight into non-perturbative corrections to heterotic orbifold compactifications.

\section{Acknowledgments}

We thank F. Apruzzi, C. Lawrie, and T. Rudelius for helpful discussions. JJH and LL thank the Banff International Research Station for hospitality during workshop 18w5190 on the Geometry and Physics of F-theory. The work of MC and LL is supported by DOE Award DE-SC0013528. MC further acknowledges the support by the Fay R. and Eugene L. Langberg Endowed Chair and the Slovenian Research Agency. The work of JJH is supported by NSF CAREER grant PHY-1756996.

\section{A Tate model with $\mathfrak{e}_{6} \times \mathfrak{s u}_{3}$}

In this appendix, we present the generic Tate model of an elliptic fibration over $B=\mathbb{F}_{6}$, whose codimension one singularities correspond to an $\mathfrak{e}_{6} \times \mathfrak{s u}_{3} \times \mathfrak{e}_{6}$ gauge algebra in F-theory. To begin with, any Weierstrass model over $\mathbb{F}_{6}$ has a non-higgsable $\mathfrak{e}_{6}$ on the -6 -curve $\{v\}$ due to the factorization

$$
f=v^{3} \tilde{f}, \quad g=v^{4} \tilde{g}
$$

where $[\tilde{f}]=5[u]+2[s]=5[v]+32[s]$ and $[\tilde{g}]=8[u]=8[v]+48[s]$. Let $\sigma=0$ denote the locus of the $\mathfrak{s u}_{3}$, with $[\sigma]=[u]$ a +6 -curve. Expanding

$$
\begin{aligned}
& \tilde{f}=f_{32} v^{5}+f_{26} v^{4} \sigma+f_{20} v^{3} \sigma^{2}+\sigma^{3} f^{\prime}, \\
& \tilde{g}=g_{48} v^{8}+g_{42} v^{7} \sigma+g_{36} v^{6} \sigma^{2}+\sigma^{3} g^{\prime},
\end{aligned}
$$

the generic way to obtain a split $\mathrm{I}_{3}$ fiber over $\sigma=0$ is to set

$$
f_{32}=-3 \phi_{8}^{4}, \quad g_{48}=2 \phi_{8}^{6}, \quad g_{42}=-\phi_{18} \phi_{8}^{3}, \quad f_{26}=\phi_{18} \phi_{8}, \quad g_{36}=\frac{\phi_{18}^{2}-12 f_{20} \phi_{8}^{2}}{12} .
$$

Next, we want to tune a split type IV fiber on another +6 -curve. Follow the $\mathfrak{e}_{7} \times \mathfrak{s u}_{2}$ example, we label this curve by $u=0$, and the $\mathfrak{s u}_{3}$ curve by $\sigma \equiv u+p_{6} v=0$ for a generic degree 6 polynomial $p_{6}$. For that, we have to tune the remaining coefficients in (A.2) 
such that $\tilde{f} \sim u^{3}$ and $\tilde{g} \sim u^{4}$. By degree counting, the general expansion for the primed functions are

$$
\begin{aligned}
& f^{\prime}=f_{2} u^{2}+f_{8} u v+f_{14} v^{2} \\
& g^{\prime}=g_{0} u^{5}+g_{6} u^{4} v+g_{12} u^{3} v^{2}+g_{18} u^{2} v^{3}+g_{24} u v^{4}+g_{30} v^{5}
\end{aligned}
$$

Inserting this into (A.2), together with $\sigma=u+p_{6} v$, we can again solve the vanishing order conditions using standard UFD methods.

The tricky part is implementing the split condition for $\mathfrak{e}_{6}$. Explicitly, this requires the following function to be a square:

$$
\left.\left(\tilde{g} / u^{4}\right)\right|_{u=0}=12 g_{6} p_{6}+12 f_{2} c_{2}^{2} p_{6}+c_{6}^{2} .
$$

The most general way to do it is by setting

$$
g_{6}=\frac{1}{12}\left(\alpha^{2} p_{6}-12 f_{2} c_{2}^{2}+2 \alpha c_{6}\right)
$$

where $\alpha$ is an arbitrary complex number. The final result of the tuning process is

$$
\begin{aligned}
f= & {\left[f_{2} u^{2}+\left(2 f_{2} p_{6}-3 c_{2}^{4}+c_{2} c_{6}\right) u v+p_{6}\left(f_{2} p_{6}-c_{2} c_{6}\right) v^{2}\right] u^{3} v^{3} } \\
g= & {\left[12 g_{0} u^{4}+\left(36 g_{0} p_{6}+2 \alpha c_{6}+\alpha^{2} p_{6}-12 f_{2} c_{2}^{2}\right) u^{3} v\right.} \\
& +\left(3\left(12 g_{0}+\alpha^{2}\right) p_{6}^{2}+24 c_{2}^{2}\left(c_{2}^{4}-f_{2} p_{6}\right)+c_{6}\left(c_{6}+12 c_{2}^{3}+6 \alpha p_{6}\right)\right) u^{2} v^{2} \\
& +\left(\left(12 g_{0}+3 \alpha^{2}\right) p_{6}^{2}+2 c_{6}\left(6 c_{2}^{3}+c_{6}\right)+6 p_{6}\left(\alpha c_{6}-2 f_{2} c_{2}^{2}\right)\right) p_{6} u v^{3} \\
& \left.+p_{6}^{2}\left(c_{6}+p_{6} \alpha\right)^{2} v^{4}\right] \frac{u^{4} v^{4}}{12} \\
\Delta= & u^{8} v^{8}\left(u+p_{6} v\right)^{3} \Delta_{\mathrm{res}}
\end{aligned}
$$

with

$$
\begin{aligned}
\Delta_{\mathrm{res}} & =27 g_{0}^{2} u^{5}+(\ldots) u^{4} v+(\ldots) u^{3} v^{2}+(\ldots) u^{2} v^{3}+(\ldots) u v^{4}+\frac{p_{6}\left(\alpha p_{6}+c_{6}\right)^{4}}{144} v^{5} \\
& =27 g_{0}^{2} s^{5}+(\ldots) s^{4} v+(\ldots) s^{3} v^{2}+(\ldots) s^{2} v^{3}+(\ldots) s v^{4}+p_{6} c_{2}^{3} Q_{18} v^{5}
\end{aligned}
$$

After blowing up the non-minimal points at $u=p_{6}=0$ and thus introducing six tensors, both the $\mathfrak{e}_{6}$ and $\mathfrak{s u}_{3}$ divisors have self-intersection 0 . Consistent with anomalies, the intersections with the residual discriminant result in six points of $\mathfrak{e}_{7}$ enhancement for $u=0$ with $\mathbf{2 7}$ matter of $\mathfrak{e}_{6}$, and 18 points of $\mathrm{I}_{4}$ enhancement for $\sigma=0$ with $\mathbf{3}$ matter of $\mathfrak{s u}_{3}$. The free parameters of the Weierstrass model (A.7) are contained in $f_{2}, c_{2}, c_{6}, p_{6}, g_{0}, \alpha$, which after subtracting $3+1$ reparametrization degree of freedoms yield 18 uncharged hypers. One can verify this counting by checking the gravitational anomaly, which is indeed canceled:

$$
\begin{aligned}
H & =6 \times 27+18 \times 3+18=234, \\
V & =2 \times 78+8=164, \\
T & =7 \\
\Longrightarrow \quad 273 & =H-V+29 T .
\end{aligned}
$$




\section{B Branching rules for bifundamental multiplets}

In this appendix we review some aspects of branching rules for representations of $\mathfrak{e}_{7} \times \mathfrak{s u}_{2}$ and $\mathfrak{e}_{6} \times \mathfrak{s u}_{3}$. This is used in the main body of the text to study the appearance of higher dimension representations of $\mathfrak{s u}_{2}$ and $\mathfrak{s u}_{3}$. All of this material is standard, and can be extracted for example from [64] as well as computer packages such as LieART [65].

\section{B.1 $\mathfrak{e}_{7} \times \mathfrak{s u}_{2}$}

To begin, we observe the following branching rules for representations of $\mathfrak{e}_{7}$ to some subalgebras. We begin with the decomposition to $\mathfrak{s o}_{12} \times \mathfrak{s u}_{2}$.

$$
\begin{aligned}
\mathfrak{e}_{7} & \supset \mathfrak{s o}_{12} \times \mathfrak{s u}_{2} \\
\mathbf{5 6} & \rightarrow(\mathbf{1 2}, \mathbf{2})+(\mathbf{3 2}, \mathbf{1}) \\
\mathbf{1 3 3} & \rightarrow(\mathbf{6 6}, \mathbf{1})+(\mathbf{3 2}, \mathbf{2})+(\mathbf{1}, \mathbf{3}) .
\end{aligned}
$$

It is also helpful to consider the decomposition of $\mathfrak{s o}_{12}$ to the subalgebra $\mathfrak{s o}_{8} \times \mathfrak{s u}_{2} \times \mathfrak{s u}_{2}$ :

$$
\begin{aligned}
\mathfrak{s o}_{12} & \supset \mathfrak{s o}_{8} \times \mathfrak{s u}_{2} \times \mathfrak{s u}_{2} \\
\mathbf{1 2} & \rightarrow\left(\mathbf{8}_{v}, \mathbf{1}, \mathbf{1}\right)+(\mathbf{1}, \mathbf{2}, \mathbf{2}) \\
\mathbf{3 2} & \rightarrow\left(\mathbf{8}_{s}, \mathbf{2}, \mathbf{1}\right)+\left(\mathbf{8}_{c}, \mathbf{1}, \mathbf{2}\right) \\
\mathbf{6 6} & \rightarrow(\mathbf{2 8}, \mathbf{1}, \mathbf{1})+\left(\mathbf{8}_{v}, \mathbf{2}, \mathbf{2}\right)+(\mathbf{1}, \mathbf{3}, \mathbf{1})+(\mathbf{1}, \mathbf{1}, \mathbf{3}),
\end{aligned}
$$

where in the above, $\boldsymbol{8}_{s}, \boldsymbol{8}_{c}$ denote the two spinor representations of $\mathfrak{s o}_{8}$.

Putting this together, we now consider the decompositions of $\mathfrak{e}_{7} \times \mathfrak{s u}_{2}$ to the subalgebra $\left(\mathfrak{s o}_{8} \times\left(\mathfrak{s u}_{2}\right)^{3}\right) \times \mathfrak{s u}_{2}$, in the obvious notation:

$$
\begin{aligned}
& \mathfrak{e}_{7} \times \mathfrak{s u}_{2} \supset \mathfrak{s o}_{8} \times\left(\mathfrak{s u}_{2}\right)^{3} \times \mathfrak{s u}_{2} \\
&(\mathbf{5 6}, \mathbf{2}) \rightarrow\left(\mathbf{8}_{v}, \mathbf{1}, \mathbf{1}, \mathbf{2}, \mathbf{2}\right)+(\mathbf{1}, \mathbf{2}, \mathbf{2}, \mathbf{2}, \mathbf{2})+\left(\mathbf{8}_{s}, \mathbf{2}, \mathbf{1}, \mathbf{1}, \mathbf{2}\right)+\left(\mathbf{8}_{c}, \mathbf{1}, \mathbf{2}, \mathbf{1}, \mathbf{2}\right) \\
&(\mathbf{5 6}, \mathbf{1}) \rightarrow\left(\mathbf{8}_{v}, \mathbf{1}, \mathbf{1}, \mathbf{2}, \mathbf{1}\right)+(1, \mathbf{2}, \mathbf{2}, \mathbf{2}, \mathbf{1})+\left(\mathbf{8}_{s}, \mathbf{2}, \mathbf{1}, \mathbf{1}, \mathbf{1}\right)+\left(\mathbf{8}_{c}, \mathbf{1}, \mathbf{2}, \mathbf{1}, \mathbf{1}\right) \\
&(\mathbf{1 3 3}, \mathbf{1}) \rightarrow(\mathbf{2 8}, \mathbf{1}, \mathbf{1}, \mathbf{1}, \mathbf{1}) \\
&+\left(\mathbf{8}_{v}, \mathbf{2}, \mathbf{2}, \mathbf{1}, \mathbf{1}\right)+\left(\mathbf{8}_{s}, \mathbf{2}, \mathbf{1}, \mathbf{2}, \mathbf{1}\right)+\left(\mathbf{8}_{c}, \mathbf{1}, \mathbf{2}, \mathbf{2}, \mathbf{1}\right) \\
&+(\mathbf{1}, \mathbf{3}, \mathbf{1}, \mathbf{1}, \mathbf{1})+(\mathbf{1}, \mathbf{1}, \mathbf{3}, \mathbf{1}, \mathbf{1})+(\mathbf{1}, \mathbf{1}, \mathbf{1}, \mathbf{3}, \mathbf{1}) \\
&(\mathbf{1}, \mathbf{2}) \rightarrow(\mathbf{1}, \mathbf{1}, \mathbf{1}, \mathbf{1}, \mathbf{2}) \\
&(\mathbf{1}, \mathbf{3}) \rightarrow(\mathbf{1}, \mathbf{1}, \mathbf{1}, \mathbf{1}, \mathbf{3}) .
\end{aligned}
$$

As per our discussion of breaking patterns in section 5 , we seek out the branching rules to the diagonal subalgebra $\mathfrak{s o}_{8} \times\left(\mathfrak{s u}_{2}\right)_{\text {diag }}$ :

$$
\begin{aligned}
& \mathfrak{e}_{7} \times \mathfrak{s u}_{2} \supset \mathfrak{s o}_{8} \times\left(\mathfrak{s u}_{2}\right)_{\mathrm{diag}} \\
&(\mathbf{5 6}, \mathbf{2}) \rightarrow\left(\mathbf{8}_{v}, \mathbf{3}\right)+\left(\mathbf{8}_{s}, \mathbf{3}\right)+\left(\mathbf{8}_{c}, \mathbf{3}\right) \\
&+\left(\mathbf{8}_{v}, \mathbf{1}\right)+\left(\mathbf{8}_{s}, \mathbf{1}\right)+\left(\mathbf{8}_{c}, \mathbf{1}\right) \\
&+(\mathbf{1}, \mathbf{5})+(\mathbf{1}, \mathbf{3})^{\oplus 3}+(\mathbf{1}, \mathbf{1})^{\oplus 2}
\end{aligned}
$$




$$
\begin{aligned}
(\mathbf{5 6}, \mathbf{1}) \rightarrow & \left(\mathbf{8}_{v}, \mathbf{2}\right)+\left(\mathbf{8}_{s}, \mathbf{2}\right)+\left(\mathbf{8}_{c}, \mathbf{2}\right)+(\mathbf{1}, \mathbf{4})+(\mathbf{1}, \mathbf{2})^{\oplus 2} \\
(\mathbf{1 3 3}, \mathbf{1}) \rightarrow & (\mathbf{2 8}, \mathbf{1}) \\
& +\left(\mathbf{8}_{v}, \mathbf{3}\right)+\left(\mathbf{8}_{s}, \mathbf{3}\right)+\left(\mathbf{8}_{c}, \mathbf{3}\right) \\
& +\left(\mathbf{8}_{v}, \mathbf{1}\right)+\left(\mathbf{8}_{s}, \mathbf{1}\right)+\left(\mathbf{8}_{c}, \mathbf{1}\right) \\
& +(\mathbf{1}, \mathbf{3})+(\mathbf{1}, \mathbf{3})+(\mathbf{1}, \mathbf{3}) \\
(\mathbf{1}, \mathbf{2}) \rightarrow & (\mathbf{1}, \mathbf{2}) \\
(\mathbf{1}, \mathbf{3}) \rightarrow & (\mathbf{1}, \mathbf{3}) .
\end{aligned}
$$

\section{B.2 $\mathfrak{e}_{6} \times \mathfrak{s u}_{3}$}

Consider now the decompositions of representations for $\mathfrak{e}_{6} \times \mathfrak{s u}$. To begin, we observe the following branching rules for representations of $\mathfrak{e}_{6}$ to some subalgebras:

$$
\begin{aligned}
\mathfrak{e}_{6} & \supset \mathfrak{s u}_{3} \times \mathfrak{s u}_{3} \times \mathfrak{s u}_{3} \\
\mathbf{2 7} & \rightarrow(\mathbf{3}, \mathbf{3}, \mathbf{1})+(\overline{\mathbf{3}}, \mathbf{1}, \mathbf{3})+(\mathbf{1}, \overline{\mathbf{3}}, \overline{\mathbf{3}}) \\
\mathbf{7 8} & \rightarrow(\mathbf{3}, \overline{\mathbf{3}}, \mathbf{3})+(\overline{\mathbf{3}}, \mathbf{3}, \overline{\mathbf{3}})+(\mathbf{8}, \mathbf{1}, \mathbf{1})+(\mathbf{1}, \mathbf{8}, \mathbf{1})+(\mathbf{1}, \mathbf{1}, \mathbf{8}) .
\end{aligned}
$$

The decomposition of the various representations of $\mathfrak{e}_{6} \times \mathfrak{s u}_{3}$ to $\left(\mathfrak{s u}_{3}\right)^{3} \times \mathfrak{s u}_{3}$ is then:

$$
\begin{aligned}
\mathfrak{e}_{6} \times \mathfrak{s u}_{3} & \supset\left(\mathfrak{s u}_{3}\right)^{3} \times \mathfrak{s u}_{3} \\
(\mathbf{2 7}, \mathbf{3}) & \rightarrow(\mathbf{3}, \mathbf{3}, \mathbf{1}, \mathbf{3})+(\overline{\mathbf{3}}, \mathbf{1}, \mathbf{3}, \mathbf{3})+(\mathbf{1}, \overline{\mathbf{3}}, \overline{\mathbf{3}}, \mathbf{3}) \\
(\mathbf{2 7}, \mathbf{1}) & \rightarrow(\mathbf{3}, \mathbf{3}, \mathbf{1}, \mathbf{1})+(\overline{\mathbf{3}}, \mathbf{1}, \mathbf{3}, \mathbf{1})+(\mathbf{1}, \overline{\mathbf{3}}, \overline{\mathbf{3}}, \mathbf{1}) \\
(\mathbf{7 8}, \mathbf{1}) & \rightarrow(\mathbf{3}, \overline{\mathbf{3}}, \mathbf{3}, \mathbf{1})+(\overline{\mathbf{3}}, \mathbf{3}, \overline{\mathbf{3}}, \mathbf{1})+(\mathbf{8}, \mathbf{1}, \mathbf{1}, \mathbf{1})+(\mathbf{1}, \mathbf{8}, \mathbf{1}, \mathbf{1})+(\mathbf{1}, \mathbf{1}, \mathbf{8}, \mathbf{1}) \\
(\mathbf{1}, \mathbf{3}) & \rightarrow(\mathbf{1}, \mathbf{1}, \mathbf{1}, \mathbf{3}) \\
(\mathbf{1}, \mathbf{8}) & \rightarrow(\mathbf{1}, \mathbf{1}, \mathbf{1}, \mathbf{8}) .
\end{aligned}
$$

We now single out the third $\left(\mathfrak{s u}_{3}\right)_{(3)}$ factor, and take the diagonal subalgebra of the first, second and fourth factors. The various representations then decompose as:

$$
\begin{aligned}
\mathfrak{e}_{6} \times \mathfrak{s u}_{3} & \supset\left(\mathfrak{s u}_{3}\right)_{(3)} \times\left(\mathfrak{s u}_{3}\right)_{\operatorname{diag}} \\
(\mathbf{2 7}, \mathbf{3}) & \rightarrow(\mathbf{1}, \mathbf{8})^{\oplus 2}+(\mathbf{1}, \mathbf{1})+(\mathbf{1}, \mathbf{1 0})+(\mathbf{3}, \mathbf{8})+(\mathbf{3}, \mathbf{1})+(\overline{\mathbf{3}}, \mathbf{8})+(\overline{\mathbf{3}}, \mathbf{1}) \\
(\mathbf{2 7}, \mathbf{1}) & \rightarrow(\mathbf{1}, 6)+(\mathbf{1}, \overline{\mathbf{3}})+(\mathbf{3}, \overline{\mathbf{3}})+(\overline{\mathbf{3}}, \overline{\mathbf{3}}) \\
(\mathbf{7 8}, \mathbf{1}) & \rightarrow(3, \mathbf{8})+(\mathbf{3}, \mathbf{1})+(\overline{\mathbf{3}}, \mathbf{8})+(\overline{\mathbf{3}}, \mathbf{1})+(\mathbf{1}, \mathbf{8})^{\oplus 2}+(\mathbf{8}, \mathbf{1}) \\
(\mathbf{1}, \mathbf{3}) & \rightarrow(\mathbf{1}, \mathbf{3}) \\
(\mathbf{1}, \mathbf{8}) & \rightarrow(\mathbf{1}, \mathbf{8}) .
\end{aligned}
$$

Open Access. This article is distributed under the terms of the Creative Commons Attribution License (CC-BY 4.0), which permits any use, distribution and reproduction in any medium, provided the original author(s) and source are credited.

\section{References}

[1] C. Vafa, The string landscape and the swampland, hep-th/0509212 [INSPIRE]. 
[2] H. Ooguri and C. Vafa, On the geometry of the string landscape and the swampland, Nucl. Phys. B 766 (2007) 21 [hep-th/0605264] [INSPIRE].

[3] C. Vafa, Evidence for F-theory, Nucl. Phys. B 469 (1996) 403 [hep-th/9602022] [InSPIRE].

[4] D.R. Morrison and C. Vafa, Compactifications of F-theory on Calabi-Yau threefolds. 1, Nucl. Phys. B 473 (1996) 74 [hep-th/9602114] [INSPIRE].

[5] D.R. Morrison and C. Vafa, Compactifications of F-theory on Calabi-Yau threefolds. 2., Nucl. Phys. B 476 (1996) 437 [hep-th/9603161] [INSPIRE].

[6] K.R. Dienes, String theory and the path to unification: A Review of recent developments, Phys. Rept. 287 (1997) 447 [hep-th/9602045] [INSPIRE].

[7] V. Kumar, D.R. Morrison and W. Taylor, Mapping $6 D N=1$ supergravities to F-theory, JHEP 02 (2010) 099 [arXiv: 0911.3393] [INSPIRE].

[8] V. Kumar, D.R. Morrison and W. Taylor, Global aspects of the space of $6 D N=1$ supergravities, JHEP 11 (2010) 118 [arXiv: 1008.1062] [INSPIRE].

[9] D.S. Park and W. Taylor, Constraints on $6 D$ supergravity theories with abelian gauge symmetry, JHEP 01 (2012) 141 [arXiv:1110.5916] [INSPIRE].

[10] L.B. Anderson, J. Gray, N. Raghuram and W. Taylor, Matter in transition, JHEP 04 (2016) 080 [arXiv: 1512.05791] [INSPIRE].

[11] M. Cvetič and L. Lin, The global gauge group structure of F-theory compactification with $\mathrm{U}(1) s$, JHEP 01 (2018) 157 [arXiv:1706.08521] [INSPIRE].

[12] D. Klevers, D.R. Morrison, N. Raghuram and W. Taylor, Exotic matter on singular divisors in F-theory, JHEP 11 (2017) 124 [arXiv:1706.08194] [INSPIRE].

[13] W. Taylor and A.P. Turner, An infinite swampland of $\mathrm{U}(1)$ charge spectra in $6 D$ supergravity theories, JHEP 06 (2018) 010 [arXiv:1803.04447] [INSPIRE].

[14] S. Gukov, M. Rangamani and E. Witten, Dibaryons, strings and branes in AdS orbifold models, JHEP 12 (1998) 025 [hep-th/9811048] [INSPIRE].

[15] T. Kobayashi et al., Stringy origin of non-Abelian discrete flavor symmetries, Nucl. Phys. B 768 (2007) 135 [hep-ph/0611020] [INSPIRE].

[16] H.P. Nilles, M. Ratz and P.K.S. Vaudrevange, Origin of family symmetries, Fortsch. Phys. 61 (2013) 493 [arXiv: 1204.2206] [INSPIRE].

[17] M. Berasaluce-Gonzalez et al., Non-abelian discrete gauge symmetries in $4 d$ string models, JHEP 09 (2012) 059 [arXiv: 1206. 2383] [INSPIRE].

[18] M. Berasaluce-Gonzalez, P.G. Camara, F. Marchesano and A.M. Uranga, $Z_{p}$ charged branes in flux compactifications, JHEP 04 (2013) 138 [arXiv: 1211.5317] [INSPIRE].

[19] V. Braun, M. Cvetič, R. Donagi and M. Poretschkin, Type II string theory on Calabi-Yau manifolds with torsion and non-Abelian discrete gauge symmetries, JHEP 07 (2017) 129 [arXiv: 1702.08071] [INSPIRE].

[20] T.W. Grimm, T.G. Pugh and D. Regalado, Non-Abelian discrete gauge symmetries in F-theory, JHEP 02 (2016) 066 [arXiv: 1504.06272] [INSPIRE].

[21] P.H. Frampton and T.W. Kephart, Simple non-Abelian finite flavor groups and fermion masses, Int. J. Mod. Phys. A 10 (1995) 4689 [hep-ph/9409330] [INSPIRE]. 
[22] L.J. Dixon, J.A. Harvey, C. Vafa and E. Witten, Strings on orbifolds, Nucl. Phys. B 261 (1985) 678 [INSPIRE].

[23] L.J. Dixon, J.A. Harvey, C. Vafa and E. Witten, Strings on orbifolds. 2, Nucl. Phys. B 274 (1986) 285 [INSPIRE].

[24] G. Honecker and M. Trapletti, Merging heterotic orbifolds and K3 compactifications with line bundles, JHEP 01 (2007) 051 [hep-th/0612030] [INSPIRE].

[25] C. Lüdeling and F. Ruehle, F-theory duals of singular heterotic K3 models, Phys. Rev. D 91 (2015) 026010 [arXiv: 1405.2928] [INSPIRE].

[26] M. Cvetič, D. Klevers, H. Piragua and W. Taylor, General U(1) $\times$ U(1) F-theory compactifications and beyond: geometry of unHiggsings and novel matter structure, JHEP 11 (2015) 204 [arXiv: 1507.05954] [INSPIRE].

[27] D. Klevers and W. Taylor, Three-index symmetric matter representations of $\mathrm{SU}(2)$ in F-theory from non-Tate form Weierstrass models, JHEP 06 (2016) 171 [arXiv: 1604.01030] [INSPIRE].

[28] N. Raghuram, Abelian F-theory models with charge-3 and charge-4 matter, JHEP 05 (2018) 050 [arXiv: 1711.03210] [INSPIRE].

[29] M. Bershadsky and A. Johansen, Colliding singularities in F-theory and phase transitions, Nucl. Phys. B 489 (1997) 122 [hep-th/9610111] [inSPIRE].

[30] P.S. Aspinwall and D.R. Morrison, Point-like instantons on K3 orbifolds, Nucl. Phys. B 503 (1997) 533 [hep-th/9705104] [INSPIRE].

[31] J.J. Heckman, D.R. Morrison and C. Vafa, On the classification of 6D SCFTs and generalized ADE orbifolds, JHEP 05 (2014) 028 [Erratum ibid. 1506 (2015) 017] [arXiv: 1312.5746] [INSPIRE].

[32] J.J. Heckman and T. Rudelius, Top down approach to 6D SCFTs, arXiv:1805.06467 [INSPIRE].

[33] M. Del Zotto, J.J. Heckman, D.R. Morrison and D.S. Park, 6D SCFTs and Gravity, JHEP 06 (2015) 158 [arXiv: 1412.6526] [INSPIRE].

[34] L.B. Anderson, A. Grassi, J. Gray and P.-K. Oehlmann, F-theory on quotient threefolds with $(2,0)$ discrete superconformal matter, JHEP 06 (2018) 098 [arXiv:1801.08658] [INSPIRE].

[35] P.S. Aspinwall and R.Y. Donagi, The Heterotic string, the tangent bundle and derived categories, Adv. Theor. Math. Phys. 2 (1998) 1041 [hep-th/9806094] [INSPIRE].

[36] L.B. Anderson, J.J. Heckman and S. Katz, T-branes and geometry, JHEP 05 (2014) 080 [arXiv: 1310.1931] [INSPIRE].

[37] L.B. Anderson, J.J. Heckman, S. Katz and L. Schaposnik, T-branes at the limits of geometry, JHEP 10 (2017) 058 [arXiv: 1702.06137] [INSPIRE].

[38] S. Cecotti, C. Cordova, J.J. Heckman and C. Vafa, T-branes and monodromy, JHEP 07 (2011) 030 [arXiv: 1010.5780] [inSPIRE].

[39] A. Collinucci and R. Savelli, F-theory on singular spaces, JHEP 09 (2015) 100 [arXiv: 1410.4867] [INSPIRE].

[40] A. Collinucci and R. Savelli, T-branes as branes within branes, JHEP 09 (2015) 161 [arXiv: 1410.4178$]$ [INSPIRE]. 
[41] I. Bena, J. Blåbäck, R. Minasian and R. Savelli, There and back again: a T-brane's tale, JHEP 11 (2016) 179 [arXiv: 1608.01221] [INSPIRE].

[42] F. Marchesano and S. Schwieger, T-branes and $\alpha^{\prime}$-corrections, JHEP 11 (2016) 123 [arXiv: 1609.02799] [INSPIRE].

[43] I. Bena, J. Blåbäck and R. Savelli, T-branes and matrix models, JHEP 06 (2017) 009 [arXiv: 1703.06106] [INSPIRE].

[44] F. Marchesano, R. Savelli and S. Schwieger, Compact T-branes, JHEP 09 (2017) 132 [arXiv: 1707.03797] [INSPIRE].

[45] M. Del Zotto, J.J. Heckman, A. Tomasiello and C. Vafa, 6D conformal matter, JHEP 02 (2015) 054 [arXiv: 1407.6359] [INSPIRE].

[46] J.J. Heckman, More on the matter of 6D SCFTs, Phys. Lett. B 747 (2015) 73 [arXiv: 1408.0006] [INSPIRE].

[47] J.J. Heckman, D.R. Morrison, T. Rudelius and C. Vafa, Atomic classification of $6 D$ SCFTs, Fortsch. Phys. 63 (2015) 468 [arXiv: 1502.05405] [INSPIRE].

[48] A. Adulpravitchai, A. Blum and M. Lindner, Non-Abelian discrete groups from the breaking of continuous flavor symmetries, JHEP 09 (2009) 018 [arXiv:0907.2332] [INSPIRE].

[49] C. Luhn, Spontaneous breaking of SU(3) to finite family symmetries: a pedestrian's approach, JHEP 03 (2011) 108 [arXiv:1101.2417] [INSPIRE].

[50] S. Katz, D.R. Morrison, S. Schäfer-Nameki and J. Sully, Tate's algorithm and F-theory, JHEP 08 (2011) 094 [arXiv: 1106. 3854] [INSPIRE].

[51] M. Bershadsky et al., Geometric singularities and enhanced gauge symmetries, Nucl. Phys. B 481 (1996) 215 [hep-th/9605200] [INSPIRE].

[52] A.P. Braun, R. Ebert, A. Hebecker and R. Valandro, Weierstrass meets Enriques, JHEP 02 (2010) 077 [arXiv: 0907.2691] [INSPIRE].

[53] D.R. Morrison and W. Taylor, Classifying bases for $6 D$ F-theory models, Central Eur. J. Phys. 10 (2012) 1072 [arXiv: 1201.1943] [INSPIRE].

[54] S.H. Katz and C. Vafa, Matter from geometry, Nucl. Phys. B 497 (1997) 146 [hep-th/9606086] [INSPIRE].

[55] V. Sadov, Generalized Green-Schwarz mechanism in F-theory, Phys. Lett. B 388 (1996) 45 [hep-th/9606008] [INSPIRE].

[56] D.R. Morrison and C. Vafa, F-theory and $\mathcal{N}=1$ SCFTs in four dimensions, JHEP 08 (2016) 070 [arXiv : 1604.03560] [INSPIRE].

[57] F. Apruzzi, J.J. Heckman, D.R. Morrison and L. Tizzano, 4D gauge theories with conformal matter, JHEP 09 (2018) 088 [arXiv:1803.00582] [INSPIRE].

[58] K. Dasgupta and S. Mukhi, F theory at constant coupling, Phys. Lett. B 385 (1996) 125 [hep-th/9606044] [INSPIRE].

[59] T.W. Grimm and T. Weigand, On abelian gauge symmetries and proton decay in global F-theory GUTs, Phys. Rev. D 82 (2010) 086009 [arXiv: 1006. 0226] [INSPIRE].

[60] D.R. Morrison and D.S. Park, F-theory and the Mordell-Weil group of elliptically-fibered Calabi-Yau threefolds, JHEP 10 (2012) 128 [arXiv:1208.2695] [INSPIRE]. 
[61] C. Mayrhofer, E. Palti and T. Weigand, U(1) symmetries in F-theory GUTs with multiple sections, JHEP 03 (2013) 098 [arXiv:1211.6742] [INSPIRE].

[62] M. Cvetič, T.W. Grimm and D. Klevers, Anomaly cancellation and abelian gauge symmetries in F-theory, JHEP 02 (2013) 101 [arXiv:1210.6034] [INSPIRE].

[63] T. Weigand, TASI Lectures on F-theory, arXiv:1806.01854 [INSPIRE].

[64] W. McKay and J. Patera, Tables of dimensions, indices, and branching rules for representations of simple Lie algebras, Lecture notes in pure and applied mathematics, M. Dekker, New York U.S.A. (1981).

[65] R. Feger and T.W. Kephart, LieART - A Mathematica application for Lie algebras and representation theory, Comput. Phys. Commun. 192 (2015) 166 [arXiv:1206.6379] [INSPIRE]. 